PREPARED FOR THE U.S. DEPARTMENT OF ENERGY, UNDER CONTRACT DE-AC02-76CH03073

PPPL-3949rev

PPPL-3949rev

UC-70

Ponderomotive Barrier

as a Maxwell Demon

by

I.Y. Dodin, N.J. Fisch, and J.M. Rax

May 2004

Revised July 2004

$N_{\substack{\text { PRInCETOn PLASMA } \\ \text { PHYSIES LABORATORY }}}^{D}$

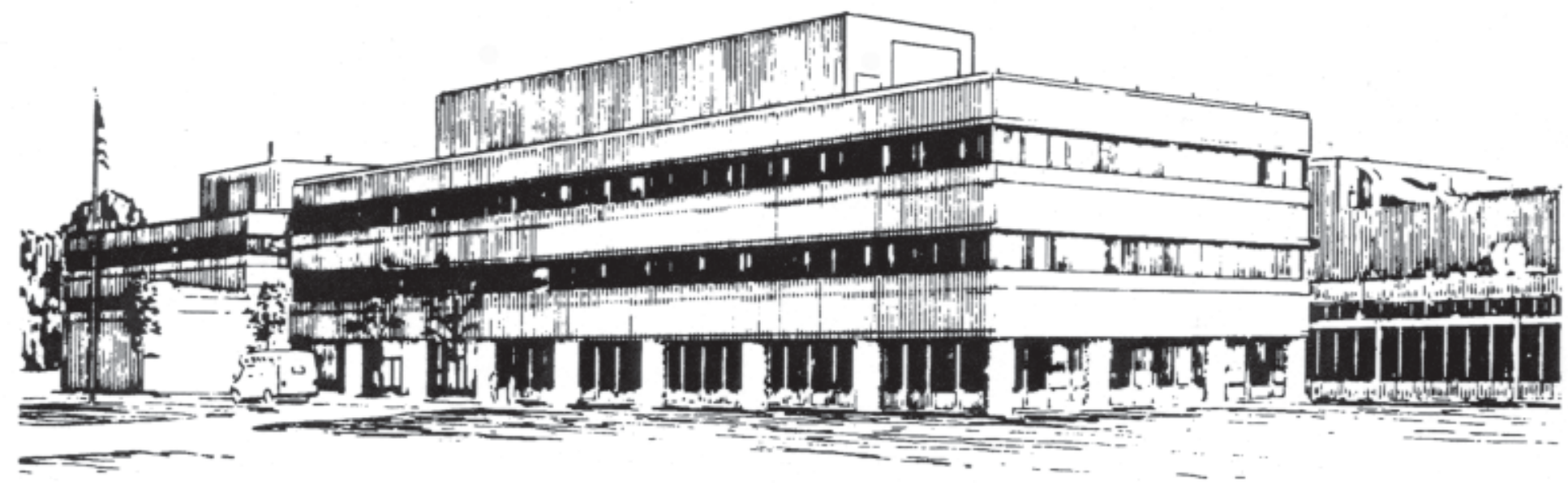

PRINCETON PLASMA PHYSICS LABORATORY PRINCETON UNIVERSITY, PRINCETON, NEW JERSEY 


\section{PPPL Reports Disclaimer}

This report was prepared as an account of work sponsored by an agency of the United States Government. Neither the United States Government nor any agency thereof, nor any of their employees, makes any warranty, express or implied, or assumes any legal liability or responsibility for the accuracy, completeness, or usefulness of any information, apparatus, product, or process disclosed, or represents that its use would not infringe privately owned rights. Reference herein to any specific commercial product, process, or service by trade name, trademark, manufacturer, or otherwise, does not necessarily constitute or imply its endorsement, recommendation, or favoring by the United States Government or any agency thereof. The views and opinions of authors expressed herein do not necessarily state or reflect those of the United States Government or any agency thereof.

\section{Availability}

This report is posted on the U.S. Department of Energy's Princeton Plasma Physics Laboratory Publications and Reports web site in Fiscal Year 2004. The home page for PPPL Reports and Publications is: http://www.pppl.gov/pub_report/

DOE and DOE Contractors can obtain copies of this report from:

U.S. Department of Energy

Office of Scientific and Technical Information

DOE Technical Information Services (DTIS)

P.O. Box 62

Oak Ridge, TN 37831

Telephone: (865) 576-8401

Fax: (865) 576-5728

Email: reports@adonis.osti.gov

This report is available to the general public from:

National Technical Information Service

U.S. Department of Commerce

5285 Port Royal Road

Springfield, VA 22161

Telephone: $1-800-553-6847$ or

(703) $605-6000$

Fax: (703) 321-8547

Internet: http://www.ntis.gov/ordering.htm 


\title{
Ponderomotive barrier as a Maxwell demon
}

\author{
I. Y. Dodin and N. J. Fisch \\ Princeton Plasma Physics Laboratory, Princeton, NJ 08543 \\ J. M. Rax \\ LPTP, Ecole Polytechnique, 91128 Palaiseau, France
}

The possibility of efficient ponderomotive current drive in a magnetized plasma was reported recently in [Phys. Rev. Lett. 91, 205004 (2003)]. Precise limitations on the efficiency are now given through a comprehensive analytical and numerical study of single-particle dynamics under the action of a cyclotron-resonant rf drive in various field configurations. Expressions for the particle energy gain and acceleration along the dc magnetic field are obtained. The fundamental correlation between the two effects is described. A second fundamental quantity, namely, the ratio of the potential barrier to the energy gain, can be changed by altering the field configuration. The asymmetric ponderomotive current drive effect can be optimized by minimizing the transverse heating.

PACS numbers: $52.35 . \mathrm{Mw}, 52.40 . \mathrm{Db}, 52.55 . \mathrm{Wq}$ 


\section{INTRODUCTION}

An intense electromagnetic field can exert a significant time-averaged force on a charged particle known as the ponderomotive, or Miller, force [1], which plays an important role in various nonlinear phenomena of waves-plasma interaction (e.g., self-focusing, filamentation, Raman scattering). In the presence of a magnetic field, ponderomotive effects are explained in terms of a pseudo-potential, which governs the particle guiding center motion along the magnetic field. The pseudo-potential can be put in the form $[1-3]$

$$
\Psi=\Phi+\mu B_{0}, \quad \Phi=\sum_{\nu} \frac{e^{2}\left|\mathbf{E}_{\mathrm{rf}, \nu}\right|^{2}}{4 m \omega(\omega+\nu \Omega)} .
$$

Here $\mathbf{E}_{\mathrm{rf}, \nu}$ is the rf field component with polarization $\boldsymbol{\tau}_{\nu}$,

$$
\boldsymbol{\tau}_{ \pm 1}=\left(\mathbf{x}^{0} \pm i \mathbf{y}^{0}\right) / \sqrt{2}, \quad \boldsymbol{\tau}_{0}=\mathbf{z}^{0}
$$

$\mathbf{x}^{0}$ and $\mathbf{y}^{0}$ are the unit vectors in the plane perpendicular to the magnetic field $\mathbf{B}_{0} \approx \mathbf{z}^{0} B_{0}(z)$, smooth on the scale of the oscillations amplitude; $\omega$ is the frequency of the rf field, and $\Omega=e B_{0} / m c$ is the Larmor frequency. The quantity $\mu=m v_{\mathrm{L}}^{2} / 2 B_{0}$ represents an approximate integral of the particle motion [2,3], analogous to the adiabatic invariant of free gyromotion in a slowly varying magnetic field. (Here $\mathbf{v}_{\mathrm{L}}=\mathbf{v}_{\perp}-\mathbf{v}_{\mathrm{rf}, \perp}$ is the velocity additional to the velocity of the rf-driven oscillations $\mathbf{v}_{\text {rf }}$ )

The ponderomotive force plays an important role in the dynamics of natural (cosmic) plasmas (see, e.g., Refs. [4-6]), yet its properties often come in useful in the laboratory as well. The practical applications include isotopes separation in plasmas composed of multiple ion species [7], as well as stabilization of low-frequency modes [8, 9] and rf plugging in magnetic confinement devices (for review, see Ref. [2]). For all of these effects, it is sufficient that drift particle dynamics follows the "adiabatic" model describable in terms of a reversible potential (1). However, for a reversible potential, it is required that $\mathrm{rf}$ and dc field profiles vary slowly compared to the particle oscillations and the beat frequency $\omega-\Omega$ changes little in a period. In a certain vicinity of the cyclotron resonance, where $\Omega(z) \approx \omega$, this condition is violated and the approximation of a ponderomotive potential (1) fails. In this case, nonadiabatic effects come into play.

As proposed in Refs. [10, 11], the ponderomotive force can be used drive electric current in a magnetized plasma through an rf barrier asymmetry: As the potential (1) experiences a singularity at the cyclotron resonance, a resonant rf field can operate essentially like a Maxwell demon (MD), reflecting particles incident on one side while transmitting those incident on the other side of the ponderomotive barrier, and hence producing a current (see Sec. II for details). Unlike a true Maxwell demon, particles experience collisionless heating while passing through the resonance, and the amount of energy they receive stochastically appears to be linked tightly with particle acceleration along the magnetic field. The asymmetric ponderomotive current drive (APCD) effect has many uses, and could be competitive with other means of rf current generation [11].

The purpose of this paper is to study the conditions under which APCD can be sustained. To explore the basics of the effect, we consider the simplest analytic model, which demonstrates the fundamental properties of the asymmetric barrier opration. In particular, we limit ourselves to the problem of nonrelativistic single-particle dynamics in given rf 
and static fields and neglect the electrostatic field, which appears in a real plasma due to charge separation caused by a ponderomotive force. In this case, for studying the current drive effect, the action of the rf field on plasma particles can be conveniently described in terms of the mapping between the particle velocities before and after the interaction with the rf field: $\mathbf{v}_{2}=\mathbf{T}\left(\mathbf{v}_{1}\right)$. In the paper, we show how the function $\mathbf{T}$ can be obtained and how these results can be used to optimize the field configurations for applications like current drive.

The nonadiabatic dynamics of rf-driven particles in the cyclotron-resonance area has been studied in a number of works $[2,5,12-19]$, primarily inspired by interest in rf plasma confinement, yet the general analytical treatment, sufficient for studying the APCD effect, has not been fully put forth. For instance, weak heating was studied for particles quasi-adiabatically trapped by an rf field within a plasma [13-15] with little attention to those transmitting through rf plugs and leaving the operating volume of the fusion device. The most general analytic model of transmitting particle dynamics was proposed in Ref. [12], where the major emphasis was made on particle acceleration from the resonance region. In other cases, effects caused by the inhomogeneity of the dc magnetic field were either studied heuristically [5], on the level of elementary estimates [2], or neglected completely [16, 17]. What remains necessary is a full and general treatment of the problem. In this paper, we present a comprehensive study of nonadiabatic particle dynamics for a broad variety of field configurations. In doing so, we address as well a specific application of current drive in a magnetized plasma.

The three main results are presented in the paper. First, we developed a nonlinear analytical model of transverse heating and longitudinal acceleration of rf-driven nonrelativistic particles near the cyclotron resonance and established integral equalities showing the fundamental correlation between the two effects. Second, we estimated the minimum rf power deposition into a plasma, which would be sufficient to sustain the asymmetry of an barrier for APCD. Third, we proposed an alternative scheme of the ponderomotive current drive of substantially higher efficiency than that recently proposed in Ref. [11], yet it remains to identify how the effect might be implemented in a plasma device in a practical manner.

The paper is organized as follows. The idea of an rf barrier operating in a quasi-MD regime is discussed and fundamental limitations on sustaining the asymmetry of the barrier are considered in Sec. II. Basic equations are introduced in Sec. III. In Sec. IV, we discuss the simplest approximations for the rf heating and the height of the ponderomotive barrier in case of smooth field profiles. In Sec. V, the transverse heating is discussed for a fairly general field configuration. In Sec. VI, we introduce the approach for calculating the average longitudinal force, which is used further in Sec. VII and VIII for particular cases of interest. In Sec. IX, we discuss how particle acceleration is connected to resonant heating and derive restrictions on the energy transfer for a realizable asymmetric rf barrier. In Sec. X, we discuss the possibility of applying a cyclotron-resonant rf barrier with reduced transverse heating for efficient current drive in plasmas, or, for that matter, for other applications as well. In Sec. XI, we present the results of our numerical calculations, and, in Sec. XII, we summarize the main results of the work. Some supplementary calculations are given in the Appendices. 


\section{ASYMMETRIC BARRIER}

Let us consider a standing wave such that the rf energy density $\mathcal{W} \equiv\left|E_{\mathrm{rf}}\right|^{2}$ is a function of $z$. If the frequency of this wave is close to the cyclotron frequency, the ponderomotive force $F_{\mathrm{pm}}$ will be quite large. Since $\mathcal{W}=0$ outside the interaction region, the gradient of $\mathcal{W}(z)$ changes sign along the particle trajectory, and the momentum transfer in the region $d \mathcal{W} / d z>0$ will be cancelled by the interaction in the region $d \mathcal{W} / d z<0$. Thus, it is impossible to put the ponderomotive force at work in a homogeneous magnetic field $B_{0}$.

However, one can design a magnetic-mirror field configuration (that is, where $B_{0}$ is nonuniform along $z$ ), such that, for the most important $\mathrm{rf}$ harmonic with the resonant circular polarization, the sign of the factor $(\omega-\Omega)^{-1}$ in $F_{\mathrm{pm}}=-d \Phi / d z$ compensates the sign of the energy density gradient $d \mathcal{W} / d z$. In the region where $d \mathcal{W} / d z>0$ the $\mathrm{rf}$ field frequency $\omega$ is below the cyclotron frequency $\Omega$, and in the region where $d \mathcal{W} / d z<0$ the rf field frequency is above $\Omega$. For example, suppose that the rf electric field envelope has a maximum at the cyclotron resonance, and thus $\mathbf{B}_{\mathrm{rf}}$ experiences phase reversal over the resonance (Fig. 1). The average Lorenz force due to rf-induced transverse particle oscillations can be put in the form $\left\langle\Delta F_{z}\right\rangle=F_{1}+F_{2}$, where

$$
F_{1}=\frac{1}{c}\left\langle\mathbf{v}_{\mathrm{rf}} \times \mathbf{B}_{0}\right\rangle_{z}, \quad F_{2}=\frac{1}{c}\left\langle\mathbf{v}_{\mathrm{rf}} \times \mathbf{B}_{\mathrm{rf}}\right\rangle_{z}
$$

To get the sign of $F_{1}$ and $F_{2}$ note that the phases of the particle oscillatory transverse displacement $\mathbf{r}_{\mathrm{rf}}(z, t)$ and the oscillatory velocity $\mathbf{v}_{\mathrm{rf}}(z, t)$ change over the resonance by $\pi$ (see Sec. III). Hence, so does the phase of the small transverse component of the dc field, $\mathbf{B}_{0, r} \approx-\frac{1}{2} \mathbf{r}_{\mathrm{rf}} B_{0, z}^{\prime}$, seen by the particle at $\mathbf{r}_{\mathrm{rf}}(z, t)$. Thus, at each $z$ both $F_{1}$ and $F_{2}$ are directed the same way, namely, towards weaker dc magnetic field. [Note also that the diamagnetic force $F_{0}$, due to a nonzero magnetic moment $\mu$ of particle Larmor rotation, $F_{0}=-\mu B_{0}^{\prime}(z)$, is similarly directed. Hence, if transversely heated by the rf field (which corresponds to an increase of $\mu$ ), a particle can also gain longitudinal acceleration by converting its perpendicular energy via a magnetic nozzle, as proposed in Ref. [20].]

This prescription permits net thrust from a standing wave [10, 11] applied near the cyclotron resonance: while particles traveling from weaker magnetic field $(\Omega<\omega)$ are decelerated and, in principle, can be reflected by the barrier, those traveling from stronger field $(\Omega>\omega)$ are automatically transmitted and accelerated by the rf field. Without resonant heating, the barrier would operate precisely like a Maxwell demon (MD), which would decrease the plasma entropy without energy deposition into the plasma and whose existence is thus prohibited by the second law of thermodynamics. Hence, the heating in principle cannot be reduced lower than a certain limit, which then determines the upper bound for a realizable current drive efficiency.

The same idea can be alternatively explained as follows. A physically realizable rf gate, which could reflect particles moving in one direction while transmitting those traveling the other way, is constrained by the Hamiltonian nature of the wave-particle interaction. Since collisionless particle motion under the action of an electromagnetic field is a Hamiltonian process, the particle phase flow remains incompressible throughout the interaction. Imagine now that we partition the particle phase space into small cells of equal volume $\Delta \Gamma_{i}=\Delta \Gamma$, and to each cell attach a certain value of the one-particle distribution function $f_{i}$. Then the number of cells that have a given value of $f$ is conserved throughout the interaction process (and so does the plasma entropy $S=-\Delta \Gamma \sum_{i} f_{i} \ln f_{i}[21]$ ), as follows from the 
Liouville theorem. Alternatively, this fact can be expressed as conservation of the so-called Casimir invariants, or Casimirs, which essentially determine the distribution of the values $f_{i}$ (see, e.g., Ref. [22]) and whose existence is an intrinsic property of any Hamiltonian system.

Suppose that plasma particles having an initial phase space distribution $f_{1}$ are introduced into an electromagnetic field for a limited time, which eventually results in bringing the plasma into some final state $f_{2}$. Since limited by the Casimirs conservation, the distribution $f_{2}$ may not be arbitrary but will rather represent a result of "restacking" of the preserved original phase-space elements $\Delta \Gamma_{i}$, regardless of the spatial and temporal structure of the field the plasma has undergone interaction with. To drive a current as efficiently as possible, one would need to minimize the energy deposition into a plasma for a given current produced by the gate. However, the fact that $f_{2}$ is a restacked $f_{1}$ imposes a limitation on the energy and momentum exchange between the rf field and plasma particles [23, 24].

Calculating the absolute limit determined by the Casimirs conservation for a gate of an arbitrary structure represents a separate problem, which we consider elsewhere (see Ref. [25]). Approaching the absolute minimum in practice is challenging (if not impossible). However, a matter of practical interest is how efficiently the power deposition can be minimized in a technically realizable gate. Here, we estimate the minimum heating, at which a realizable rf barrier remains capable of driving a current, and propose a near-optimum current drive scheme. First we discuss the single-particle dynamics in the vicinity of a cyclotron resonance, to which the next few sections are devoted.

\section{BASIC EQUATIONS}

To explore the basics of the APCD effect, let us consider the simplest model, which demonstrates the fundamental properties of the asymmetric barrier operation. Let us assume that the motion of a particle (an ion or an electron) can be adequately described by nonrelativistic equations. Let us assume also that the rf radiation represents a transverse plane wave of an arbitrary longitudinal structure, while the influence of the electrostatic field, which can appear in a real plasma due to charge separation caused by the ponderomotive force, is of minor importance. In this case, the particle motion is governed by the equation

$$
m \frac{d \mathbf{v}}{d t}=e\left(\mathbf{E}_{\mathrm{rf}}+\frac{\mathbf{v}}{c} \times\left(\mathbf{B}_{\mathrm{rf}}+\mathbf{B}_{0}\right)\right)
$$

Assume a linearly polarized localized rf field (for alternative polarizations, see Appendix A) determined by the vector potential (in units $m c^{2} / e$ )

$$
\mathbf{A}_{\mathrm{rf}}=\mathbf{x}^{0} \varepsilon(z) \cos \tau, \quad \varepsilon( \pm \infty)=0
$$

and thus given by

$$
\mathbf{E}_{\mathrm{rf}}=\mathbf{x}^{0} \varepsilon(z) \sin \tau, \quad \mathbf{B}_{\mathrm{rf}}=\mathbf{y}^{0} \varepsilon^{\prime}(z) \cos \tau
$$

in units $m \omega c / e$. Here $\omega$ is the frequency of the field, $\tau=\omega t$ is the dimensionless time, and $z$ is the longitudinal coordinate. The normalized rf field amplitude $\varepsilon$ is assumed small compared to unity, as it represents the ratio of the 
momentum imparted by the wave field in a single oscillation to $m c$. Consider also a dc magnetic field determined by the vector potential, which we approximate by a linear function with respect to the transverse displacement:

$$
\mathbf{A}_{0}(\mathbf{r})=\frac{1}{2} b(z)\left(\mathbf{z}^{0} \times \mathbf{r}\right)
$$

(in units $m c^{2} / e$ ). Then, in units $m \omega c / e$, the actual field $\mathbf{B}_{0}=\nabla \times \mathbf{A}_{0}$ can be written as

$$
\mathbf{B}_{0}=-\frac{x b^{\prime}}{2} \mathbf{x}^{0}-\frac{y b^{\prime}}{2} \mathbf{y}^{0}+b \mathbf{z}^{0}
$$

where the particle coordinates are measured in units $c / \omega$. The dimensionless function $b(z)$ is approximately equal to the magnetic field strength measured in units $m \omega c / e$, or the local ratio of the nonrelativistic Larmor frequency $\Omega=e B_{0} / m c$ normalized on $\omega$. Thus, $b(z)=1$ at the cyclotron resonance located at $z=0$.

In dimensionless notation, when the particle velocity is normalized on the speed of light $c$, the scalar motion equations can be put in the following form:

$$
\begin{aligned}
& \dot{v}_{x}=v_{y} b+y v_{z} \frac{b^{\prime}}{2}+\varepsilon \sin \tau-v_{z} \varepsilon^{\prime} \cos \tau \\
& \dot{v}_{y}=-v_{x} b-x v_{z} \frac{b^{\prime}}{2} \\
& \dot{v}_{z}=v_{x} \varepsilon^{\prime} \cos \tau+\left(x v_{y}-y v_{x}\right) \frac{b^{\prime}}{2} .
\end{aligned}
$$

The two types of oscillations are intrinsic to the particle motion in rf and dc magnetic fields. Those are free Larmor rotation with frequency $b(z)$ and rf-driven oscillations with unit frequency (in dimensionless notation). If the relative change in the beat frequency between the two is small over a period of that frequency and the spatial scale of the rf field $L_{E}$ is large enough, i.e.,

$$
\frac{v_{z}}{(1-b)^{2}} \frac{d(1-b)}{d z} \ll 1, \quad L_{E} \gg \frac{v_{z}}{|1-b|}
$$

then the two types of oscillations uncouple [3, 13], and one can solve for the transverse particle motion to get

$$
\mathbf{r}_{\perp}=\mathbf{r}_{\mathrm{d}}+\mathbf{r}_{\mathrm{rf}}, \quad \mathbf{v}_{\perp}=\mathbf{v}_{\mathrm{d}}+\mathbf{v}_{\mathrm{rf}}
$$

Here $\mathbf{r}_{\mathrm{d}}$ and $\mathbf{v}_{\mathrm{d}}$ are the drift displacement and velocity undergoing free Larmor oscillations, while $\mathbf{r}_{\mathrm{rf}}$ and $\mathbf{v}_{\mathrm{rf}}$ stand for rf-driven (to the leading order, purely transverse) velocity and displacement given by

$$
\begin{array}{rlrl}
x_{\mathrm{rf}} & =-\frac{\varepsilon \sin \tau}{1-b^{2}}, & y_{\mathrm{rf}}=-\frac{\varepsilon b \cos \tau}{1-b^{2}}, \\
v_{\mathrm{rf}, x}=-\frac{\varepsilon \cos \tau}{1-b^{2}}, & v_{\mathrm{rf}, y}=\frac{\varepsilon b \sin \tau}{1-b^{2}} .
\end{array}
$$

Under the approximation of smooth rf and dc fields (10), two integrals of particle motion are approximately conserved [1-3]: the magnetic moment of the drift motion

$$
\mu=\frac{v_{\mathrm{d}}^{2}}{2 b}
$$

and the so-called quasi-energy of the longitudinal motion

$$
\mathfrak{E}=\frac{v_{z}^{2}}{2}+\mu b+\Phi
$$


where the ponderomotive potential $\Phi$ is given by

$$
\Phi(z)=\frac{\varepsilon^{2}(z)}{4\left(1-b^{2}(z)\right)} .
$$

In the vicinity of the cyclotron resonance $(b(z) \approx 1)$, the average potential $\Phi(z)$ yields a singularity, which is a clear sign of the ponderomotive approximation failure. To describe particle motion in this regime, more detailed analysis is needed.

\section{QUASI-LINEAR APPROXIMATION FOR SMOOTH FIELDS}

First, consider the simplest case when the spatial scale of the dc magnetic field is large compared to the particle longitudinal gyroradius:

$$
\Lambda=L_{B} / v_{z} \gg 1
$$

assuming $L_{E} / v_{z} \gg 1$ as well. Suppose the rf field amplitude is approximately constant across the resonance region, $\varepsilon(z) \approx \varepsilon_{0}$, while the magnetic field strength can be approximated with a linear function

$$
b(z)=1+z / L_{B}
$$

(Fig. 2). Then the dominant heating effect can be calculated as follows. Consider the general expression for the average energy change

$$
\langle\Delta \mathcal{E}\rangle=\left\langle\int_{-\infty}^{\infty} v_{x} \varepsilon(z(\tau)) \sin \tau d \tau\right\rangle
$$

with expression for the particle velocity $v_{x}$ given by Eq. (12), which, in dimensional complex notation, is given by

$$
v_{x}=\frac{i \omega}{\omega^{2}-\Omega^{2}} \frac{e E_{x}}{m} e^{-i \omega t}
$$

Noting that the linear (in $\varepsilon$ ) response function $v_{x}(\omega)$ cannot experience a singularity in the upper half of the $\omega$ complex plane, when integrating across the cyclotron resonance, one must shift the singularity from the real axis correspondingly $[21,26]$ to get

$$
\langle\Delta \mathcal{E}\rangle=\frac{1}{2} \Im \int_{-\infty}^{\infty} \frac{\varepsilon(z(\tau))^{2}}{b(z(\tau))^{2}-1-i 0} d \tau
$$

With $d z=v_{z} d \tau$ and assuming $v_{z}=$ const in the resonance region, one obtains

$$
\langle\Delta \mathcal{E}\rangle=\frac{\pi}{4} \varepsilon_{0}^{2} \Lambda
$$

The assumption of constant $v_{z}$ and linear response $v_{x}(\omega)$ to calculate the nonadiabatic heating effect constitute the so-called quasi-linear approximation, which is valid only for fast particles. Indeed, as $v_{z}$ goes to zero, the expression (21) experiences a singularity being a sign of the quasi-linear approximation failure. Hence, for low velocities, detailed 
analysis is needed to calculate $\langle\Delta \mathcal{E}\rangle$ more accurately, namely, by considering the finite width of the region where a particle undergoes resonant interaction with the rf field. The width of this region [13]

$$
\bar{z}=\sqrt{L_{B} v_{z}}
$$

can be readily obtained from Eq. (10), which yields the condition $|z| \gg \bar{z}$ for the reversible quasi-potential (15) to be established. The time scale $\bar{\tau}$, over which the particle passes the resonance region, is then of the order of $\sqrt{\Lambda}$. Therefore, a fast particle does not have sufficient time for being heated, and thus, energy gain must decrease as its longitudinal velocity is increased - exactly as predicted by Eq. (21). However, this scaling holds only for magnetic fields (17) smooth inside the interaction region, unlike abrupt fields, for which the conclusion must be revised (Sec. VIII).

The effective height of the barrier, which determines the maximum longitudinal energy a particle must have to be reflected adiabatically, can be estimated as $\Phi_{\max } \sim \Phi(\bar{z})$, or

$$
\Phi_{\max } \sim \varepsilon^{2} \sqrt{\Lambda}
$$

and thus depends on the particle initial longitudinal velocity [15]. The characteristic of the barrier independent of the particle initial velocity is the "critical" energy $\hat{\mathcal{E}}=\varepsilon_{0}^{8 / 5} L_{B}^{2 / 5}$, or the critical velocity $\hat{v}=\sqrt{\hat{\mathcal{E}}}$,

$$
\hat{v}=\varepsilon_{0}^{4 / 5} L_{B}^{1 / 5}
$$

Particles with $v_{z} \ll \hat{v}$ are reflected adiabatically from the barrier if moving from the weak-field side $(\Omega<\omega)$. Those which travel in the same direction with $v_{z} \gtrsim \hat{v}$ (and all of those traveling backwards and hence experiencing ponderomotive acceleration rather than deceleration) penetrate the resonance region and undergo cyclotron heating. (In this case, the quantity $\hat{v}$ equals the characteristic momentum a particle gains inside the resonance region [12].) For particles having $v_{z} \gg \hat{v}$ and traveling each way, the longitudinal velocity change is small compared to $v_{z}$. For more accurate description of transverse heating and longitudinal acceleration, a general model of particle nonadiabatic dynamics is developed in the next sections.

It must be understood that the presented results remain applicable only for classical particle motion. Even small relativistic modification of the Larmor frequency can change the scalings, which describe the resonant interaction of a particle with an rf field (see, e.g., Ref. [12]). The characteristic time, at which the particle ceases to interact resonantly with the field because of relativistic shift of the gyrofrequency can be estimated as $\tau_{\text {rel }} \sim \varepsilon^{-2 / 3}$. If $\tau_{\text {rel }} \lesssim \bar{\tau}$, relativistic effects become dominant, and the spatial scale $L_{B}$ no longer determines the amount of energy a particle can gain from the rf field. However, if $\tau_{\text {rel }} \gg \bar{\tau}$, relativistic corrections remain of minor importance, and the assumption of classical particle dynamics (used in this paper) becomes sufficient for adequate description of the APCD effect. For velocities $v_{z} \sim \hat{v}$, the latter condition, which we will assume satisfied, can be expressed in terms of the rf field amplitude $\varepsilon_{0}$ and the characteristic scale of the dc field inhomogeneity $L_{B}$ :

$$
\varepsilon_{0}^{4 / 15} L_{B}^{2 / 5} \ll 1 .
$$




\section{TRANSVERSE HEATING}

To solve for the transverse particle motion, let us first introduce a complex transverse displacement

$$
\rho=x+i y=r e^{i \phi},
$$

in terms of which the transverse motion equation takes the following form:

$$
\ddot{\rho}+i b \dot{\rho}+\frac{i}{2} \rho \dot{b}=-\frac{d}{d \tau}(\varepsilon \cos \tau) .
$$

For given functions $b(\tau)$ and $\varepsilon(\tau)$, this equation turns to a linear ODE, so that one can write

$$
\begin{aligned}
& \rho(\tau)=\rho_{\mathrm{f}}(\tau)+\rho_{\mathrm{rf}}(\tau), \\
& \rho_{\mathrm{f}}\left(\tau_{0}\right)=\rho_{0}, \\
& \rho_{\mathrm{rf}}\left(\tau_{0}\right)=0,
\end{aligned}
$$

where $\rho_{\mathrm{f}}$ stands for the solution of a homogeneous equation (i.e., describes free Larmor oscillations with initial displacement $\rho_{0}$ ), while $\rho_{\text {rf }}$ stands for the rf-driven oscillations and vanish if $\varepsilon \equiv 0$.

In the region of the most efficient interaction with the rf field, $\rho_{\mathrm{rf}}$ and $\rho_{\mathrm{f}}$ oscillate at approximately equal (unit) frequencies, and the particle orbit is approximately circular. The latter is also true away from the rf field, where the gyroradius is inverse proportional to $b$. Then, in the leading-order approximation, one can accept the general formula

$$
\rho=i w / b
$$

where we introduced a complex velocity $w=\dot{\rho}$,

$$
w=v_{x}+i v_{y}
$$

Hence, one can rewrite Eq. (27) as a first-order ODE for $w$ :

$$
\dot{w}+i b w-\frac{\dot{b}}{2 b} w=-\frac{d}{d \tau}(\varepsilon \cos \tau) .
$$

Supposing the motion starts at $\tau=\tau_{0}$ at the location $z=z_{0}$, one can get an exact solution of this equation:

$$
\begin{aligned}
& w=w_{\mathrm{f}}+w_{\mathrm{rf}}, \\
& w_{\mathrm{f}}=w_{0} \sqrt{\frac{b(z(\tau))}{b\left(z_{0}\right)}} \exp \left(-i \int_{\tau_{0}}^{\tau} b\left(z\left(\tau^{\prime}\right)\right) d \tau^{\prime}\right),
\end{aligned}
$$

where $w_{0}=v_{x, 0}+i v_{y, 0}$ is the initial value of the complex velocity, and the rf-driven part of $w$ is given by

$$
w_{\mathrm{rf}}=-\int_{-\infty}^{\tau} \sqrt{\frac{b(z(\tau))}{b\left(z\left(\tau^{\prime}\right)\right)}} \frac{d}{d \tau^{\prime}}\left(\varepsilon\left(z\left(\tau^{\prime}\right)\right) \cos \tau^{\prime}\right) \times \exp \left(-i \int_{\tau}^{\tau^{\prime}} b\left(z\left(\tau^{\prime \prime}\right)\right) d \tau^{\prime \prime}\right) d \tau^{\prime} .
$$

Near the cyclotron resonance, in the leading-order approximation one can take $b \approx 1$ and replace the full-time derivative under the integral with $f_{1}=-\varepsilon \sin \tau^{\prime}$, as the remaining term $f_{2}=\dot{\varepsilon} \cos \tau^{\prime}$ is relativistic and represents the 
higher-order correction. Indeed, the term $f_{2}$ could contribute significantly to $w_{\mathrm{rf}}$ in abrupt fields, where the magnetic rf field is large compared to the electric rf component. However, in the worst case of a delta-shaped $\varepsilon^{\prime}(z)$, taking a nonzero $f_{2}$ into account would yield only a small correction $\delta w_{\mathrm{rf}} \sim \varepsilon$, which is much less than $w_{\mathrm{rf}}$ as can be seen from the following estimate. Let us put Eq. (34) in the form

$$
w_{\mathrm{rf}}=\int_{-\infty}^{\tau} \varepsilon\left(z\left(\tau^{\prime}\right)\right) \exp \left(-i \int_{\tau}^{\tau^{\prime}} b\left(z\left(\tau^{\prime \prime}\right)\right) d \tau^{\prime \prime}\right) \sin \tau^{\prime} d \tau^{\prime} .
$$

To estimate $w_{\text {rf }}$, suppose the simplest case when $v_{z}$ changes insignificantly during the interaction, the rf field is approximately uniform across the resonance region, and the dc magnetic field changes linearly in the vicinity of the resonance (Sec. IV). Applying the steepest descent method to the integral (35), one gets for $\tau \rightarrow \infty$ :

$$
\left|w_{\mathrm{rf}}\right|=\varepsilon \sqrt{\frac{\pi L_{B}}{2 v_{z}}}
$$

[in compliance with Eq. (21)], or

$$
w_{\mathrm{rf}} \sim \varepsilon \sqrt{\Lambda}
$$

Despite in abrupt fields the expressions (22), (36) are invalid, the scalings (23), (37) yet hold (see Sec. VIII) if $\Lambda$ is defined according to

$$
\Lambda \sim \frac{1}{(1-b(\bar{z}))^{2}} \gg 1
$$

where $\bar{z}$ stands for the edge of the nonadiabatic region and can be obtained from Eq. (10). Further, we accept Eq. (38) as the general definition of $\Lambda$.

Now consider $\tau\left(z, \tau_{0}\right)$ as the moment of time when a particle arrives at a given location $z$ if launched at the moment $\tau_{0}$ from the given location $z_{0}$ :

$$
\tau\left(z, \tau_{0}\right)=\tau_{0}+\int_{z_{0}}^{z} \frac{d z^{\prime}}{v_{z}\left(z^{\prime}, \tau_{0}\right)}
$$

(Note that, generally, $v_{z}$ may represent a multivalued function, and the integration should be performed over the particle trajectory including all the branches of $v_{z}(z)$.) Assuming (39), let us rewrite Eq. (35) as

$$
w_{\mathrm{rf}}=\frac{1}{2 i}\left(h_{+} e^{-i \chi_{+}+i \tau}-h_{-} e^{-i \chi_{-}-i \tau}\right)
$$

where we introduced the quantities

$$
\begin{aligned}
& h_{ \pm}\left(z, \tau_{0}\right)=\int_{z_{0}}^{z} \varepsilon\left(z^{\prime}\right) e^{i \chi_{ \pm}\left(z^{\prime}, \tau_{0}\right)} \frac{d z^{\prime}}{v_{z}\left(z^{\prime}, \tau_{0}\right)} \\
& \chi_{ \pm}\left(z, \tau_{0}\right)=\int_{z_{0}}^{z}\left(b\left(z^{\prime}\right) \pm 1\right) \frac{d z^{\prime}}{v_{z}\left(z^{\prime}, \tau_{0}\right)} .
\end{aligned}
$$

The functions $h_{ \pm}$approximate the complex amplitudes of the rf-driven velocities of opposite circular polarizations, and thus $h_{-} \gg h_{+}$, as the rf field pumps up primarily the cyclotron-resonant component of the particle velocity. More 
precisely, the functions scale like $h_{ \pm} \sim \varepsilon /(1 \pm b)$, meaning that

$$
h_{-} / h_{+}=\mathcal{O}(\sqrt{\Lambda})
$$

In terms of $h_{ \pm}$, the expression for the rf-produced energy gain $\mathcal{E}_{\mathrm{rf}}=\frac{1}{2}\left|w_{\mathrm{rf}}\right|^{2}$ can be put in the form

$$
\mathcal{E}_{\text {rf }}=\frac{1}{8}\left(\left|h_{+}\right|^{2}+\left|h_{-}\right|^{2}\right)+\frac{1}{8}\left(h_{+} h_{-}^{*} e^{2 i \tau_{0}}+\text { c.c. }\right) \text {, }
$$

or, taking Eq. (42) into account,

$$
\mathcal{E}_{\mathrm{rf}}=\frac{|h|^{2}}{8}, \quad h \equiv h_{-} .
$$

Then, to the leading-order approximation, one can estimate the rf-produced change of the particle magnetic moment $\Delta \mu_{\mathrm{rf}} \equiv\left\langle\mathcal{M}_{\mathrm{rf}}\right\rangle(\infty)$ with the following expression:

$$
\Delta \mu_{\mathrm{rf}} \approx \frac{1}{8}\left\langle|H|^{2}\right\rangle
$$

where $H \equiv h(z(\infty))$,

$$
H\left(\tau_{0}\right)=\int \varepsilon(z) e^{i \chi\left(z, \tau_{0}\right)} \frac{d z}{v_{z}\left(z, \tau_{0}\right)}
$$

and $\chi \equiv \chi_{-}$. As seen from the derivation, the expression holds for arbitrary (both smooth and abrupt) profiles of the dc magnetic field and the rf field. Therefore, studying the properties of $H$ considered as a functional of the field profiles $\varepsilon(z), b(z)$ and the particle trajectory $z\left(\tau, \tau_{0}\right)$ can yield a complete information about the particle transverse heating under fairly general conditions.

Thereby, let us ask a question if it is possible to adjust $\varepsilon(z)$ and $b(z)$ to minimize transverse heating for the majority of particles, whose longitudinal velocities vary in a wide range $\Delta v_{z} \sim v_{z}$. To answer that, let us first rewrite the expression for $H$ in the following form:

$$
H=i \int V(z) \frac{d e^{i \chi}}{d z} d z, \quad V(z)=\frac{\varepsilon(z)}{1-b(z)} .
$$

To figure out the physical meaning of the function $V(z)$, note that the rf-driven oscillations of the particle transverse velocity in the adiabatic regime (12) can be expressed as

$$
\mathbf{v}_{\mathrm{rf}}(z, \tau) \approx \frac{1}{2} V(z) \mathbf{u}(\tau)
$$

where $\mathbf{u}=(-\cos \tau, \sin \tau)$ depends entirely on $\tau$. Thus, $V(z)$ can be thought of as the amplitude of adiabatic rf-driven oscillations. One can see that if $V(z)$ is smooth compared to $e^{i \chi}$ everywhere along the particle trajectory (which, for transmitting particles, requires also that $\varepsilon(z)$ must be equal to zero at the resonance), the integral

$$
H=-i \int e^{i \chi} d V
$$

is exponentially small irrespectively of $\mathbf{v}_{0}$. Though trivial for adiabatically reflected particles, this statement is important when applied to those which penetrate the resonance region, as it predicts that a reversible ponderomotive barrier can be formed even for transmitting particles. 
Hence, to ensure that the transverse heating becomes small for all particles irrespectively of their velocity, the amplitude of adiabatic rf-driven oscillations $V(z)$ must change little in a period of these oscillations. In other words, one must have

$$
\frac{\varepsilon(z)}{1-b(z)} \approx \mathrm{const}
$$

within the whole region of resonant interaction, yet the functions $\varepsilon(z)$ and $b(z)$ are not required to be constant or even continuous by themselves (see Sec. VIII).

\section{AVERAGE LONGITUDINAL FORCE: GENERAL APPROACH}

Now let us calculate the average force accelerating a particle in the direction parallel to the dc magnetic field. In this section, we will present the general approach to this problem, while the calculation of the longitudinal force for particular cases of interest will be given in Sec. VII, VIII (see also Appendix A).

Under the conditions of adiabatic approximation (10), the longitudinal force $\mathcal{F}_{z}=\dot{v}_{z}$ can be obtained by differentiating Eq. (14):

$$
\mathcal{F}_{z}=-\frac{d}{d z}(\mu b+\Phi)
$$

However, this expression does not adequately describe the particle motion near the cyclotron resonance, where $\Phi$ experiences a singularity. However, even in this case, under certain conditions, one can also derive a relatively simple expression for $\mathcal{F}_{z}$, from which fundamental qualitative conclusions can be made regarding the longitudinal acceleration. Consider the full longitudinal force $\mathcal{F}_{z}=\mathcal{F}_{\varepsilon}+\mathcal{F}_{b}$ where the force applied to a particle from the rf field is $\mathcal{F}_{\varepsilon}=v_{x} \varepsilon^{\prime} \cos \tau$, and the one applied from the dc magnetic field is $\mathcal{F}_{b}=\frac{1}{2}\left(x v_{y}-y v_{x}\right) b^{\prime}$. Let us put $\mathcal{F}_{z}$ in the form

$$
\mathcal{F}_{z}=\frac{d U}{d z}-\varepsilon \frac{d}{d z}\left(v_{x} \cos \tau\right)+\left(x v_{y}-y v_{x}\right) \frac{b^{\prime}}{2},
$$

where $d / d z$ stands for the full derivative

$$
\frac{d}{d z}=\frac{\partial}{\partial z}+\frac{1}{v_{z}} \frac{\partial}{\partial t}
$$

and $U$ is given by $U=\varepsilon v_{x} \cos \tau$. In terms of $\rho$ and $w$, one gets

$$
\mathcal{F}_{b}=-\frac{b^{\prime}}{2} \Im\left(\rho w^{*}\right)=-\mathcal{M} b^{\prime}, \quad \mathcal{M}=-\frac{r^{2} \dot{\phi}}{2},
$$

where $\mathcal{M}$ is the magnetic moment of the particle. In a uniform magnetic field without an $\operatorname{rf}$ drive, one has $\mathcal{M}=v_{\perp}^{2} / 2 b$, which also yields a good approximation for $\mathcal{M}$ if the local motion is only slightly perturbed from circular by an rf field or a dc field inhomogeneity. (Note that in smooth fields, when $\varepsilon(z)$ has a maximum at the cyclotron resonance, one has $\mathcal{F}_{\varepsilon} \ll \mathcal{F}_{b}$ in the resonance area, hence a particle is accelerated toward weaker dc field at all z.)

Using Eq. (28), one gets for $\mathcal{M}$ averaged over initial gyrophases $\phi_{0}$ :

$$
\langle\mathcal{M}\rangle_{\phi_{0}}=\left\langle\mathcal{M}_{\mathrm{f}}\right\rangle_{\phi_{0}}+\left\langle\mathcal{M}_{\mathrm{rf}}\right\rangle_{\phi_{0}},
$$


where $\mathcal{M}_{\mathrm{f}}$ stands for the magnetic moment of free Larmor oscillations determined by the particle initial velocity, and $\mathcal{M}_{\mathrm{rf}}$ is the rf-produced part of the total magnetic moment. Then, if averaged over the initial gyrophase $\phi_{0}$, the longitudinal force (52) applied to a particle at given $z$ can then be written as

$$
\left\langle\mathcal{F}_{z}\right\rangle=\left\langle\mathcal{F}_{0}\right\rangle+\left\langle\mathcal{F}_{\text {rf }}\right\rangle+\left\langle\Delta \mathcal{F}_{1}\right\rangle+\left\langle\Delta \mathcal{F}_{2}\right\rangle
$$

where we omitted the $\phi_{0}$ subindex for clarity and introduced the following quantities:

$$
\begin{aligned}
& \left\langle\mathcal{F}_{0}\right\rangle=-\left\langle\mathcal{M}_{\mathrm{f}}\right\rangle b^{\prime}, \\
& \left\langle\mathcal{F}_{\mathrm{rf}}\right\rangle=\frac{d}{d z}\left(\langle U\rangle+(1-b)\left\langle\mathcal{M}_{\mathrm{rf}}\right\rangle\right), \\
& \left\langle\Delta \mathcal{F}_{1}\right\rangle=-\varepsilon \frac{d}{d z}\left\langle v_{x} \cos \tau\right\rangle, \\
& \left\langle\Delta \mathcal{F}_{2}\right\rangle=(b-1) \frac{d\left\langle\mathcal{M}_{\mathrm{rf}}\right\rangle}{d z} .
\end{aligned}
$$

The force $\left\langle\mathcal{F}_{0}\right\rangle$ is the one a particle would "feel" in the same magnetic field without the rf drive. The other terms stand for rf-driven forces to be calculated below.

First, let us estimate the order of those. Assuming $\mathcal{M}_{\mathrm{rf}} \sim \varepsilon^{2} \Lambda$ (see Sec. V), one has each of the terms to be of the same order. However, while the diamagnetic force proportional to the change of $\mathcal{M}_{\mathrm{rf}}$ continues to accelerate a particle in a nonuniform dc magnetic field even away from the $\mathrm{rf}$ field, the terms $\left\langle\Delta \mathcal{F}_{1}\right\rangle$ and $\left\langle\Delta \mathcal{F}_{2}\right\rangle$ disappear as $\varepsilon$ goes to zero. To ensure that the expression for the total force will allow calculating the particle longitudinal energy change

$$
\left\langle\Delta \mathcal{E}_{||}\right\rangle=\int\left\langle\mathcal{F}_{z}\right\rangle d z
$$

with an error small compared to $\Phi \sim \varepsilon^{2} \sqrt{\Lambda}$, one may evaluate the forces $\left\langle\Delta \mathcal{F}_{1}\right\rangle$ and $\left\langle\Delta \mathcal{F}_{2}\right\rangle$ in the leading order with respect to $\Lambda$ using Eq. (35) instead of Eq. (34).

Let us put the expressions for $\left\langle\Delta \mathcal{F}_{1}\right\rangle$ and $\left\langle\Delta \mathcal{F}_{2}\right\rangle$ in the following form:

$$
\left\langle\Delta \mathcal{F}_{1}\right\rangle=-\varepsilon \Re G^{\prime}, \quad\left\langle\Delta \mathcal{F}_{2}\right\rangle=(b-1) \mathcal{E}_{\mathrm{rf}}^{\prime},
$$

where $G=\left\langle w_{\mathrm{rf}} \cos \tau\right\rangle$, and where we took into account that it is only the rf-driven term that contributes to the $G$. Using the results obtained in Sec. V, one gets immediately

$$
\left\langle\Delta \mathcal{F}_{2}\right\rangle=-(1-b) \frac{d}{d z} \frac{|h|^{2}}{8} .
$$

Obtaining a simple formula for $\left\langle\Delta \mathcal{F}_{1}\right\rangle$, in turn, requires rewriting the expression for $G$,

$$
G=\frac{h_{+} e^{-i \chi_{+}}}{4 i}\left(1+e^{2 i \tau}\right)-\frac{h_{-} e^{-i \chi_{-}}}{4 i}\left(1+e^{-2 i \tau}\right) .
$$

Making certain assumptions about the fields structure allows to simplify Eq. (61) and proceed with derivation, as we do below. 


\section{SMOOTH FIELDS}

In smooth fields where the spatial scales of a dc field and an rf field far exceed the particle longitudinal gyroradius $\left(L_{B} / v_{z} \gg 1, L_{E} / v_{z} \gg 1\right)$, one can average the longitudinal force over the oscillation period. As the condition (16) provides that the number of oscillations within the resonance region $N \sim \sqrt{\Lambda}$ is also large compared to unity, such averaging can be performed not only in the adiabatic region, but near the cyclotron resonance as well.

By averaging Eq. (61) over the fast particle oscillations, one gets

$$
\varepsilon\left\langle G^{\prime}\right\rangle=\frac{v_{z}}{4 i}\left(\left|h_{+}^{\prime}\right|^{2}-\left|h_{-}^{\prime}\right|^{2}\right)-\frac{1}{4}(1+b) h_{+} h_{+}^{\prime *}-\frac{1}{4}(1-b) h_{-} h_{-}^{\prime *}
$$

where we took into account that

$$
\frac{\varepsilon}{v_{z}} e^{-i \chi_{ \pm}}=\frac{d h_{ \pm}^{*}}{d z}
$$

and denoted averaging over $\tau$ with angle brackets. Thus, using Eq. (42), one can write approximately

$$
\left\langle\Delta \mathcal{F}_{1}\right\rangle=(1-b) \frac{d}{d z} \frac{|h|^{2}}{8}
$$

so that

$$
\left\langle\Delta \mathcal{F}_{1}\right\rangle+\left\langle\Delta \mathcal{F}_{2}\right\rangle=0
$$

Finally, noting that in smooth fields $\mu_{0} \equiv\left\langle\mathcal{M}_{\mathrm{f}}\right\rangle=$ const, one can formulate the obtained results as follows: In smooth fields, the average of the longitudinal force over the fast particle oscillations and the initial gyrophase $\phi_{0}$ simultaneously is given by

$$
\begin{aligned}
& \left\langle\mathcal{F}_{z}\right\rangle=\frac{d}{d z}\left(\langle U\rangle+(1-b)\left\langle\mathcal{M}_{\mathrm{rf}}\right\rangle-\mu_{0} b\right), \\
& \langle U\rangle=-\frac{v_{z}}{4} \Im\left(h \frac{d h^{*}}{d z}\right) .
\end{aligned}
$$

First, note that the obtained expressions reproduce the adiabatic model for smooth fields outside the resonance, as in the limit (10) one has

$$
\langle U\rangle=-\frac{\varepsilon^{2}}{4(1-b)}, \quad\left\langle\mathcal{M}_{\mathrm{rf}}\right\rangle=\frac{\varepsilon^{2}}{8(1-b)^{2}}
$$

so that the ponderomotive force (51) is recovered with the potential (15) evaluated in the limit $|1-b| \ll 1$. However, an important result can be obtained from Eq. (66) for nonadiabatic motion as well. Indeed, the advantage of the representation $(66)$ is that $\left\langle\mathcal{F}_{z}\right\rangle$ can be easily integrated along the particle trajectory with known boundary conditions

$$
\begin{gathered}
\langle U\rangle( \pm \infty)=0, \quad\left\langle\mathcal{M}_{\mathrm{rf}}\right\rangle(-\infty)=0 \\
\left\langle\mathcal{M}_{\mathrm{rf}}\right\rangle(+\infty)=\langle\Delta \mu\rangle
\end{gathered}
$$

to obtain the net longitudinal energy change (58) as a function of the transverse heating:

$$
\left\langle\Delta \mathcal{E}_{||}\right\rangle=\langle\Delta \mu\rangle\left(1-b_{0}\right)
$$


For the transverse and the total particle energy changes, one then gets

$$
\left\langle\Delta \mathcal{E}_{\perp}\right\rangle=\langle\Delta \mu\rangle b_{0}, \quad\langle\Delta \mathcal{E}\rangle=\langle\Delta \mu\rangle
$$

where the change of the particle magnetic moment yields $\langle\Delta \mu\rangle=\Delta \mu_{\mathrm{rf}}$ and is given by Eq. (45).

The formulas (70), (71) are analogous to the known result $\langle\Delta \mathcal{E}\rangle=\langle\Delta \mu\rangle$ for smoothly inhomogeneous fields (below we will show that this result has a broader region of applicability), easily derivable (see, e.g., Ref. [27]) if the dc magnetic field local inhomogeneity is totally neglected $(\Lambda=\infty)$. The new result, however, is the accuracy of Eq. (70). As follows from our analysis, the error of Eq. (70) is of the order of $\varepsilon^{2}$, which is much less than $\Phi_{\max }$ even for finite $\Lambda$. Hence, in case when the condition (50) is satisfied, that is in the case of zero transverse heating, particles cannot be accelerated along the magnetic field: the average longitudinal momentum of the order of $\hat{v}$, which they gain while being accelerated adiabatically, is compensated by the momentum gained by particles inside the resonance region. The consequence of this effect will be discussed in the next sections.

\section{ABRUPT FIELDS}

Let us now consider the opposite case of abrupt fields, where, even if the phase-dependent velocity modulation by the rf wave $\left(\partial v_{z} / \partial \tau_{0}\right)$ is substantial, the region of nonadiabatic motion is yet short enough to provide that the longitudinal phase modulation is negligible:

$$
\frac{\partial \tau\left(z, \tau_{0}\right)}{\partial \tau_{0}}-1=\frac{\partial}{\partial \tau_{0}} \int \frac{d z}{v_{z}\left(z, \tau_{0}\right)} \ll 1
$$

where the integral is taken over the resonance region. This condition allows us to accept a random rf phase approximation (RPA) for particles entering the resonance region with a given longitudinal velocity, under which a simple expression for $\mathcal{F}_{z}$ can be obtained by averaging over the particle ensemble.

To proceed, let us also accept the "equivalent path approximation" (EPA), by which we will mean that all particles with a given initial velocity follow the same path, irrespectively of their initial rf phases $\tau_{0}$ (and $\phi_{0}$, as also implicitly assumed before), yet $z(\tau)$ may be different for different particles. In this case, one can introduce a force acting on an average particle by averaging over $\tau_{0}$ and $\phi_{0}$ the true longitudinal force $\mathcal{F}_{z}\left(z, \tau_{0}, \phi_{0}\right)$, which particles experience at given location $z$. While $\left\langle\mathcal{F}_{\text {rf }}\right\rangle$ and $\left\langle\Delta \mathcal{F}_{2}\right\rangle$ are averaged over $\tau_{0}$ straightforwardly, it remains to perform the averaging of $\left\langle\Delta \mathcal{F}_{1}\right\rangle$, for which one gets

$$
\left\langle\Delta \mathcal{F}_{1}\right\rangle=(1-b) \frac{d}{d z} \frac{|h|^{2}}{8}-\varepsilon \Re\left\langle\delta G^{\prime}\right\rangle
$$

with the rapidly oscillating part of $G$ given by

$$
\delta G=\frac{1}{4 i}\left(h_{+} e^{-i \chi_{+}+2 i \tau}-h_{-} e^{-i \chi_{-}-2 i \tau}\right) .
$$

Expressing $h_{ \pm}$in terms of $\chi_{ \pm}$,

$$
h_{ \pm}\left(z, \tau_{0}\right)=-i \int_{z_{0}}^{z} \frac{\varepsilon\left(z^{\prime}\right)}{b\left(z^{\prime}\right) \pm 1} \frac{d}{d z^{\prime}} e^{i \chi_{ \pm}\left(z^{\prime}, \tau_{0}\right)} d z^{\prime}
$$


one can see that $\langle\delta G\rangle_{\tau_{0}} \approx 0$ under the RPA. Hence, under the valid RPA and EPA in abrupt fields, the longitudinal force averaged simultaneously over the initial $\mathrm{rf}$ phase $\tau_{0}$ and the initial gyrophase $\phi_{0}$ is given by

$$
\left\langle\mathcal{F}_{z}\right\rangle=\left\langle\mathcal{F}_{0}\right\rangle+\frac{d}{d z}\left(\langle U\rangle+(1-b)\left\langle\mathcal{M}_{\mathrm{rf}}\right\rangle\right)
$$

with $\langle U\rangle$ again given by Eq. (67). Note that the force $\left\langle\mathcal{F}_{0}\right\rangle$ no longer remains conservative in this case, since in abrupt magnetic field $\mu$ is not a conserved quantity even in the absence of the rf drive.

The RPA condition within the resonance region does not prevent from inertial bunching of particles behind it. It means that the equality (76) can be violated after the particles have undergone a complete transition through the resonance region. However, in the smooth fields behind the resonance, Eq. (66) becomes valid for the $\phi_{0}$-averaged force applied to particles launched at each particular $\tau_{0}$. Let us assume again that particles start and finish their motion in regions with the same magnetic field $b=b_{0}$. Then, averaging the longitudinal force both over $\tau_{0}$ and $\phi_{0}$ and integrating over the whole trajectory, one gets

$$
\left\langle\Delta \mathcal{E}_{||}\right\rangle=\Delta \mu_{\mathrm{rf}}\left(1-b_{0}\right)-\Delta \mu_{b} b_{0}
$$

where we introduced the quantity

$$
\Delta \mu_{b}=-\frac{1}{b_{0}} \int\left\langle\mathcal{F}_{0}\right\rangle d z .
$$

Outside the rf field, the particle magnetic moment $\mathcal{M}$ matches $\mu$, and thus

$$
\langle\Delta \mu\rangle=\Delta\left\langle\mathcal{M}_{\mathrm{f}}\right\rangle+\Delta \mu_{\mathrm{rf}}
$$

The average transverse energy change is then given by

$$
\left\langle\Delta \mathcal{E}_{\perp}\right\rangle=\Delta\left\langle\mathcal{M}_{\mathrm{f}}\right\rangle b_{0}+\Delta \mu_{\mathrm{rf}} b_{0}
$$

and the total energy change yields

$$
\langle\Delta \mathcal{E}\rangle=\Delta \mu_{\mathrm{rf}}+\left(\Delta\left\langle\mathcal{M}_{\mathrm{f}}\right\rangle-\Delta \mu_{b}\right) b_{0}
$$

For a given trajectory $z(\tau), \Delta\left\langle\mathcal{M}_{\mathrm{f}}\right\rangle$ does not depend on the rf field strength. Thus, from the fact that $\Delta \mathcal{E}=0$ at $\varepsilon=0$ (as the dc magnetic field does not change the particle energy), it follows that $\Delta \mu_{b}=\Delta\left\langle\mathcal{M}_{\mathrm{f}}\right\rangle$. Therefore, $\Delta \mu_{b}$ stands for the particle magnetic moment change caused by abrupt inhomogeneities of the dc magnetic field. Finally, the longitudinal, the transverse, and the total particle energy changes can be expressed as

$$
\begin{aligned}
& \left\langle\Delta \mathcal{E}_{||}\right\rangle=\langle\Delta \mu\rangle\left(1-b_{0}\right)-\Delta \mu_{b}, \\
& \left\langle\Delta \mathcal{E}_{\perp}\right\rangle=\langle\Delta \mu\rangle b_{0}, \\
& \langle\Delta \mathcal{E}\rangle=\Delta \mu_{\mathrm{rf}},
\end{aligned}
$$

where the change of the particle magnetic moment is given by

$$
\langle\Delta \mu\rangle=\Delta \mu_{b}+\Delta \mu_{\mathrm{rf}},
$$


with $\Delta \mu_{b}$ accounting for the dc field inhomogeneity, and $\Delta \mu_{\mathrm{rf}}$ accounting for the rf heating. Note that the previously obtained Eqs. (70), (71) can be considered as a special case of Eqs. (82) with $\Delta \mu_{b}=0$ - just as one could expect for a smooth field configuration.

To calculate $\Delta \mu_{\mathrm{rf}}$ for a case of an abrupt field, suppose that the particle motion remains adiabatic along the whole trajectory except for a short (compared to the oscillation period) time when the particle crosses a single "boundary" at $z=0$, where the rf field and the dc magnetic field have a discontinuity:

$$
\begin{gathered}
\varepsilon(z)=\varepsilon_{1}+\theta(z)\left(\varepsilon_{2}-\varepsilon_{1}\right), \\
b(z)=b_{1}+\theta(z)\left(b_{2}-b_{1}\right) .
\end{gathered}
$$

Here $\theta$ is the Heaviside's step-function, $b_{1,2} \lessgtr 1$ (or vice versa), and

$$
\Lambda \sim \frac{1}{\left(1-b_{1}\right)^{2}} \sim \frac{1}{\left(1-b_{2}\right)^{2}}
$$

is a large number. Then, from Eqs. (45), (46), it follows that

$$
\Delta \mu_{\mathrm{rf}} \approx \frac{1}{8}\left(\frac{\varepsilon_{1}}{1-b_{1}}-\frac{\varepsilon_{2}}{1-b_{2}}\right)^{2} .
$$

As one can see from Eq. (86), heating does not vanish even in the limit when the time of crossing the resonance region is infinitesimally small, which results from a nonzero leap of the oscillatory velocity [ $\left.\mathbf{v}_{\mathrm{rf}}\right]$ over the resonance. Indeed, to the leading order, one has

$$
\left[\mathbf{v}_{\mathrm{d}}\right]=[\mathbf{v}]-\left[\mathbf{v}_{\mathrm{rf}}\right] \approx \mathbf{v}_{\mathrm{rf}, 1}-\mathbf{v}_{\mathrm{rf}, 2}
$$

so that, generaly, the obtained rf-caused change of $\mu, \Delta \mu_{\mathrm{rf}} \approx \frac{1}{2}\left\langle\left[\mathbf{v}_{\mathrm{d}}\right]^{2}\right\rangle$, is of the order of $\varepsilon^{2} \Lambda$, unless the ratio of the rf field amplitudes $\varepsilon_{1} / \varepsilon_{2}$ is appropriately chosen to reduce the heating. However, if

$$
\frac{\varepsilon_{1}}{\varepsilon_{2}} \approx \frac{1-b_{1}}{1-b_{2}}
$$

$\Delta \mu_{\mathrm{rf}}$ becomes of the order of $\varepsilon^{2}$, in accordance with Eq. (50) obtained under more general consideration.

One can also come to Eqs. (82), (86) using an alternative approach, which we will further show to yield more results of interest, and which is hence worth considering in details here. Applying the quasi-energy and $\mu$ conservation laws to the particle motion before and after crossing the resonance, one gets

$$
\begin{aligned}
& \mathcal{E}_{||, 0}+\mu_{0} b_{0}=\mathcal{E}_{||, 1}+\mu_{0} b_{1}+\Phi_{1}, \\
& \mathcal{E}_{||, 2}+\mu_{2} b_{2}+\Phi_{2}=\mathcal{E}_{||, 3}+\mu_{2} b_{0},
\end{aligned}
$$

where $\mu_{2}=\mu_{0}+\Delta \mu$, and $\Phi_{1,2}$ are the values of the ponderomotive potential on each side of the resonance. As the particle crosses the resonance, its total energy is approximately conserved:

$$
\mathcal{E}_{\|, 1}+\frac{v_{\perp, 1}^{2}}{2}=\mathcal{E}_{\|, 2}+\frac{v_{\perp, 2}^{2}}{2}
$$

where $\mathbf{v}_{\perp}$ stands for the total transverse velocity, including both the drift and the oscillatory velocities. However, each of the components of $\mathbf{v}$ experiences a finite change when the particle crosses the resonance, as the transverse 
magnetic field (both $\mathrm{rf}$ and $\mathrm{dc}$ ) is $\delta$-shaped for abrupt $\varepsilon(z)$ and $b(z)$. To calculate the transverse velocity change for transmitting particles, consider the motion equations (9) from where one gets (assuming $[\mathbf{r}]=0)$ :

$$
\begin{aligned}
& {\left[v_{x}\right]=\left(\varepsilon_{1}-\varepsilon_{2}\right) \cos \tau-y\left(b_{1}-b_{2}\right) / 2,} \\
& {\left[v_{y}\right]=x\left(b_{1}-b_{2}\right) / 2 .}
\end{aligned}
$$

Using the above expressions, one can calculate the change of the particle magnetic moment (83) averaged over the initial cyclotron and rf phases:

$$
\begin{aligned}
& \Delta \mu_{b}=\Psi \frac{\left(b_{1}-b_{2}\right)^{2}}{8 b_{1} b_{2}}, \\
& \Delta \mu_{\mathrm{rf}} \approx \frac{1}{2}\left(\mathbf{v}_{\mathrm{rf}, 1}-\mathbf{v}_{\mathrm{rf}, 2}\right)^{2},
\end{aligned}
$$

where $\Psi=\left\langle r_{\mathrm{d}, 1}^{2}\right\rangle b_{1}$, and $\mathbf{r}_{\mathrm{d}, 1}$ is the particle drift displacement just before crossing the resonance.

Further, from the quasi-energy conservation (89), it follows that

$$
\Delta \mathcal{E}_{\|}=\Delta \mu\left(1-b_{0}\right)+Q
$$

where $\Delta \mathcal{E}_{\|} \equiv \mathcal{E}_{\|, 3}-\mathcal{E}_{\|, 0}$ and

$$
Q=\left[\Phi-\frac{v_{\perp}^{2}}{2}-\mu(1-b)\right] .
$$

Now substitute Eqs. (83) and (91) into the above expression to get

$$
\langle Q\rangle=-\Delta \mu_{b}+\mathcal{O}\left(\varepsilon^{2}\right)
$$

Finally, we obtain for the change of the transmitting particle longitudinal energy:

$$
\left\langle\Delta \mathcal{E}_{||}\right\rangle=\Delta \mu_{\mathrm{rf}}\left(1-b_{0}\right)-\Delta \mu_{b} b_{0}+\mathcal{O}\left(\varepsilon^{2}\right)
$$

while the transverse and the total energy changes are given by

$$
\left\langle\Delta \mathcal{E}_{\perp}\right\rangle=\langle\Delta \mu\rangle b_{0}, \quad\langle\Delta \mathcal{E}\rangle=\Delta \mu_{\mathrm{rf}}+\mathcal{O}\left(\varepsilon^{2}\right)
$$

Note that similar expressions can be derived for reflecting particles as well. Indeed, for a reflecting particle one has $\left[\mathbf{v}_{\perp}\right]=0$, as seen from Eqs. (91), where one should take $b_{2}=b_{1}$, as the particle eventually returns to the initial magnetic field. (In more details, particle longitudinal dynamics in abrupt fields is discussed in Appendix B and Sec. X.) Then, $\Delta \mu=0$, and $\left\langle\Delta \mathcal{E}_{||}\right\rangle=\left\langle\Delta \mathcal{E}_{\perp}\right\rangle=\langle\Delta \mathcal{E}\rangle=0$, in compiance with Eqs. (97), (98).

The main results we have presented up to this point are formulated in Eqs. (45)-(46) and Eqs. (82), (83), valid for both transmitted and reflected particles in case of smooth and abrupt fields with arbitrary rf polarization (see also Appendix A). The application of these results to the ponderomotive current drive problem constitutes the subject of the second part of the paper. 


\section{HEATING VS. ACCELERATION}

Let us show how our results give the fundamental constraints on the extent, to which the asymmetry of the ponderomotive barrier can be sustained. Consider particles traveling from the weak-field side of the barrier $(\Omega<\omega$, or, for clarity, $z<0$ ) with initial longitudinal velocity $v_{z}=v_{z, 0}$ and magnetic moment $\mu=\mu_{0}$. Suppose these particles are reflected adiabatically at some point $z_{\mathrm{r}}<0$, so that for each $z$ within the adiabatic region for which $z_{\mathrm{r}}<z<0$, the following condition is satisfied:

$$
\mathcal{E}_{||, 0}+\mu_{0}\left(b_{0}-b(z)\right)-\Phi(z)<0
$$

where $\mathcal{E}_{\|, 0}$ is the initial longitudinal energy, and $b_{0} \equiv b( \pm \infty)$. Consider now particles traveling from the strong-field side $(\Omega>\omega$, or $z>0)$ with initial longitudinal velocity $v_{z}=-v_{z, 0}$ and the same initial magnetic moment $\mu=\mu_{0}$. Suppose these particles transit through the barrier and penetrate the region of adiabatic motion at $z<0$ with the longitudinal energy change given by

$$
\Delta \mathcal{E}_{\| \mid}=\Delta \mu\left(1-b_{0}\right)+Q
$$

Combining the results of Sec. VII, VIII (and Appendix A), we interpret $Q$ as any nonadiabatic contribution, or "longitudinal heating", provided by abrupt variations of a dc magnetic field or an rf field. Using the quasi-energy conservation together with the above equality, one gets:

$$
\mathcal{E}_{||}+\left(\mu_{0}+\Delta \mu\right) b(z)+\Phi(z)=\mathcal{E}_{\mid l, 0}+\Delta \mu+\mu_{0} b_{0}+Q
$$

Since $\mathcal{E}_{\|}$is positively defined, one thus may note that the particle cannot be found at any location $z<0$, for which the following sufficient condition is satisfied:

$$
\mathcal{E}_{||, 0}+\mu_{0}\left(b_{0}-b(z)\right)-\Phi(z)<\Delta \mu(b(z)-1)-Q
$$

If $\Delta \mu=0$ and $Q=0$, the conditions (99) and (102) coincide. Therefore, at any $z$ within the adiabatic region for which $z_{\mathrm{r}}<z<0$, there can be no particles transmitted through the barrier in this case.

To ensure that particles traveling from the weak-field side do not penetrate further than up to $z_{\mathrm{r}}$, while those symmetrically traveling from the opposite side may be found at $z>z_{\mathrm{r}}$ after crossing the resonance, the following condition must be satisfied:

$$
\Delta \mu(b(z)-1)-Q<W(z)<0
$$

where $W$ stands for the left-hand side of Eqs. (99) and (102). The necessary condition for the obtained inequality to be possible is

$$
\Delta \mu>-\frac{Q}{1-b}
$$

as motion at the weak-field side $(b<1)$ is considered. 
Suppose now that the RPA and the EPA are valid, so that the average rf longitudinal heating is zero in compliance with Eqs. (82a), (96). Then, for an average particle to be transmitted, one must have

$$
\langle\Delta \mu\rangle>\frac{\Delta \mu_{b}}{1-b}
$$

which means, at least, that $\langle\Delta \mu\rangle>0$. Hence, under the RPA and the EPA for smooth and abrupt field profiles of arbitrary polarization, where the average rf-driven longitudinal heating is always zero, the following statement is true: If particles with initial longitudinal velocity $v_{z, 0}$ and initial magnetic moment $\mu_{0}$ are adiabatically reflected from the barrier, then particles with initial longitudinal velocity $-v_{z, 0}$ and the same initial magnetic moment will also be reflected from the barrier, unless they experience finite rf heating. In other words, a heating-free barrier is symmetric: If one makes all the particles from one side to reflect adiabatically, then, to ensure that particles traveling from the other side are not reflected, one must heat them. A proper adjustment of the field structure [see Eq. (50)] could reduce heating, but such an adjustment would automatically ruin the asymmetry of the barrier. Namely, in this case, the average momentum imparted by the adiabatic force would be compensated by the average momentum nonadiabatically gained by particles within the resonance region. This fact remains in agreement with the argument given in Sec. II, where we predicted from the first principles that a heating-free barrier cannot produce a current.

Let us estimate the minimum heating required for sustaining the asymmetry of a barrier. Suppose that a barrier adiabatically reflects particles with longitudinal energies $\mathcal{E}_{\|, 0}<\mathcal{E}_{*}$, while particles traveling backwards with the same initial parameters are transmitted. Then, for all $\mathcal{E}_{\|, 0}$, one has $z_{\mathrm{r}}\left(\mathcal{E}_{\|, 0}\right)<z_{*}$, where $z_{*} \equiv z_{\mathrm{r}}\left(\mathcal{E}_{*}\right)<0$. A necessary condition for particles traveling from the strong-field side to appear at $z_{*}$ is

$$
\mathcal{E}_{||, 0}+\mu_{0}\left(b_{0}-b\left(z_{*}\right)\right)-\Phi\left(z_{*}\right)>\Delta \mu\left(b\left(z_{*}\right)-1\right)-Q
$$

which can also be put as follows:

$$
\Delta \mu\left(b\left(z_{*}\right)-1\right)-Q<\Phi\left(z_{\mathrm{r}}\right)-\Phi\left(z_{*}\right)+W\left(z_{\mathrm{r}}\right)
$$

where we neglected the term $\mu_{0}\left(b\left(z_{\mathrm{r}}\right)-b\left(z_{*}\right)\right)=\mathcal{O}\left(\varepsilon^{2}\right)$. By definition of $z_{\mathrm{r}}$, one has $W\left(z_{\mathrm{r}}\right)=0$ and $b\left(z_{*}\right)<1$, so that the above condition takes the form

$$
\Delta \mu>\frac{\Phi\left(z_{*}\right)-\Phi\left(z_{\mathrm{r}}\right)-Q}{1-b\left(z_{*}\right)}
$$

Under the RPA and the EPA, one has $\langle Q\rangle=0$ in case of negligible $\Delta \mu_{b}$. Then, taking for the majority of particles $z_{*}-z_{\mathrm{r}}$ of the order of $\bar{z}$, one obtains the following restriction on the minimum transverse heating:

$$
\langle\Delta \mu\rangle=\mathcal{O}\left(\varepsilon^{2} \Lambda\right)
$$

Although Eq. (109) is a necessary condition, the obtained scaling simultaneously coincides with the maximum possible $\langle\Delta \mu\rangle$ an rf barrier can yield (see Sec. V). Thus, Eq. (109) represents also a sufficient condition for sustaining the asymmetry of an rf barrier with zero average longitudinal rf heating.

The only way to avoid strong transverse heating while having an asymmetric a barrier is to provide that $Q>$ $\Phi\left(z_{*}\right)-\Phi\left(z_{\mathrm{r}}\right)$, as Eq. (108) allows reducing $\Delta \mu$ down to zero in this case. Thus, in principle, an rf barrier can 
transmit particles incident from the strong-field side, which experience primarily longitudinal heating

$$
Q=\mathcal{O}\left(\varepsilon^{2} \sqrt{\Lambda}\right)>0
$$

while particles traveling backwards with the same initial energy and magnetic moment are reflected adiabatically. For obtaining the current drive effect, the condition (110) must be satisfied in average over particles interacting with the rf field. As under the RPA and the EPA, Eq. (110) cannot be satisfied, one of the two assumptions must be violated. In Sec. X, we show how the EPA can be broken in an abrupt-field configuration and propose a concept of the reduced-heating current drive scheme based on this method.

\section{ACCELERATION VIA LONGITUDINAL HEATING}

To see how a nonzero average heating can be achieved in a simple field geometry, consider an abrupt-field configuration (84), where

$$
b_{1}=1 \mp \frac{\alpha}{\sqrt{\Lambda}}, \quad b_{2}=1 \pm \frac{1}{\sqrt{\Lambda}}
$$

$(\alpha>0)$, and the rf field satisfies the reduced-heating condition (50): $\varepsilon_{1}=-\alpha \varepsilon_{2}$ (Fig. 3). Applying the analysis similar to that given in Sec. V, one gets for $\phi_{0}$-averaged quantities:

$$
\begin{aligned}
& \langle\Delta \mu\rangle_{\phi_{0}}=\Delta \mu_{b}+\mathcal{O}\left(\varepsilon^{2}\right), \\
& \langle Q\rangle_{\phi_{0}}= \pm \frac{1+\alpha}{4 \alpha} \hat{v}^{2} \cos 2 \hat{\tau}-\Delta \mu_{b}+\mathcal{O}\left(\varepsilon^{2}\right),
\end{aligned}
$$

where $\hat{v}^{2}=\left|\varepsilon_{1} \varepsilon_{2}\right| \sqrt{\Lambda}$. The moment $\hat{\tau}$, at which a particle passes the resonance, is given by

$$
\hat{\tau}=\tau_{0}+\int_{z_{0}}^{0} \frac{d z}{v_{z}(z)},
$$

where the second term is approximately $\tau_{0}$-idependent, as particles move adiabatically before reaching the "boundary" $(z=0)$.

Assume $\mu_{0}=0$ and neglect $\Delta \mu_{b}$ for clarity. All particles incident from the weak-field side are reflected by the ponderomotive force at $z<0$ if $v_{z, 0}<\frac{1}{2} \hat{v}$. Also, if $\beta<-\cos 2 \hat{\tau}$, where

$$
\beta=\frac{2 \alpha}{1+\alpha}\left(\frac{v_{z, 0}}{\hat{v}}\right)^{2}
$$

a particle is reflected nonadiabatically, while its transverse energy is still conserved. While the energy of a reflected particle is preserved, the longitudinal energy of a transmitted particle after the interaction with the rf field is changed according to

$$
\frac{\Delta \mathcal{E}_{\| \mid}(\hat{\tau})}{\mathcal{E}_{\|, 0}}= \pm \frac{1}{\beta} \cos 2 \hat{\tau}
$$

$\left(v_{z, 0} \lessgtr 0\right)$, as follows from Eq. (113). If $v_{z, 0}>\frac{1}{2} \hat{v}$ and $\beta>-\cos 2 \hat{\tau}$, a particle traveling from the weak-field side is transmitted automatically. To derive the transmission condition for a particle traveling from the strong-field side, 
note that a substantial contribution into the energy change (116) is provided by the reversible ponderomotive force already after the particle has passed the nonadiabatic region. In other words, after a transmitted particle leaves the rf field, its longitudinal energy is always larger than $\Phi_{1}$, yet the particle velocity immediately after crossing the boundary is given by

$$
\frac{\left(v_{z}\right)_{z=0-}}{v_{z, 0}}=\sqrt{1-\frac{1}{\beta}\left(\cos 2 \hat{\tau}+\frac{\alpha}{2(1+\alpha)}\right)} .
$$

Thus, the transmission takes place if

$$
\beta>\frac{\alpha}{2(1+\alpha)}+\cos 2 \hat{\tau}
$$

and no particles are reflected on the strong-field side if

$$
\beta>\frac{3 \alpha+2}{2(1+\alpha)}
$$

The above calculation shows that, in abrupt fields, the EPA is violated for sufficiently low velocities. Hence, the proposed configuration can operate as an asymmetric barrier, which adiabatically reflects all particles incident from the weak-field side with $\mathcal{E}_{\|, 0}<\frac{1}{8} \hat{v}^{2}$, while transmitting [with $\Delta \mathcal{E}_{\|}$given by Eq. (116)] some of those traveling backwards without substantial transverse heating. The outcome of the wave-plasma interaction will be an uncompensated current of transmitted particles through the resonance region, as those which are reflected (on any side) carry no current whatsoever. The fraction $\mathcal{R}$ of reflected particles with a given initial velocity among those, which are incident from the strong-field side, can be written as

$$
\mathcal{R}=\frac{1}{2 \pi} \int_{0}^{2 \pi} \theta\left(\cos 2 \hat{\tau}+\frac{\alpha}{2(1+\alpha)}-\beta\right) d \hat{\tau}
$$

under the assumption of uniform distribution of initial phases. Hence, the transmission coefficient $\mathcal{T}=1-\mathcal{R}$ equals

$$
\mathcal{T}=\frac{1}{\pi} \arccos \left(\frac{\alpha}{2(1+\alpha)}-\beta\right) .
$$

For $\beta<\frac{\alpha}{2(1+\alpha)}$ (which matches the condition of adiabatic reflection of particles symmetrically incident from the weak-field side), one gets

$$
\frac{1}{\pi} \arccos \left(\frac{\alpha}{2(1+\alpha)}\right) \leq \mathcal{T} \leq \frac{1}{2}
$$

from where it follows that $\frac{1}{3} \leq \mathcal{T} \leq \frac{1}{2}$. For $\alpha=1$, Eq. (122) yields $0.42<\mathcal{T}<0.5$, which means that, roughly, a half of all particles incident from the strong-field side is transmitted.

Let us now address the question if the current drive can be practiced alternatively through violation of the RPA (rather than the EPA) in more complicated field configurations. To break the RPA, one needs the inertial phase modulation within the resonance region

$$
\Delta \tau=\Delta\left(\frac{\bar{z}}{v_{z}}\right) \sim \frac{\Delta v_{z}}{v_{z}} \bar{\tau},
$$

to be of the order of unity, where $\Delta v_{z}$ stands for the phase-dependent rf velocity modulation, and $\bar{\tau}=\bar{z} / v_{z}$ is the time a particle needs to pass the resonance region. In plasmas with thermal velocity spread $\delta v_{z} \sim v_{\text {th }}$, phase mixing 
will depress the average longitudinal heating if $\bar{\tau} \gg 1$, hence $\bar{\tau} \lesssim 1$ is necessary. Thus, for efficient particle bunching within the resonance region one needs $\Delta v_{z} / v_{z} \gtrsim 1$, which, in turn, requires $v_{\mathrm{th}} \sim \hat{v}$. As the phase-dependent part of the longitudinal force can both accelerate and decelerate particles (as we showed above), a significant part of those traveling from the strong-field side will be reflected from the barrier in this case. It means that the violation of the RPA in a plasma would automatically lead to the violation of the EPA, which will make the current drive scheme similar to the one proposed above.

What we have shown here is that by abruptly modifying the phase of the electric fields, it is possible to heat electrons longitudinally with an energy gain proportional to $\sqrt{\Lambda}$, rather than in transverse direction with an energy gain proportional to $\Lambda$. Since $\Lambda \gg 1$ is contemplated, the rearrangement in velocity space with less heating has three advantages: First, it can avoid relativistic effects (which limit the current drive efficiency particular for longitudinal heating). Second, by limiting the energy of the affected particles it keeps the distribution function closer to Maxwellian, thereby leaving less free energy for unwanted instabilities. And, three, heating particles in the longitudinal rather than the transverse direction allows avoiding production of the unwanted Ohkawa current in toroidal plasmas [28], which otherwise is generated by transversely heated particles trapping in local "magnetic mirrors" formed because of the system geometry.

One can compare to LHCD [29] and to ECCD [30] or to other means of driving rf currents [31]. In both LCCD and ECCD, electrons are moved to higher energy, thereby becoming less collisional and thus realizing greater current before slowing down. In the case of the less efficient asymmetric ponderomotive current drive [11], the effect of reflection (which is most efficient) was mitigated by being averaged with the effects of current drive through heating longitudinally (LCCD) or transversely (ECCD). In the case of the optimized APCD, the reflection current is accomplished, but the mitigation effects are less, because there is less heating as described above.

The ability to change fields abruptly will be easier in reflecting ions rather than electrons, because of the larger ion gyroradius. In particular, the minority species current drive effect [32] operates similarly to the ECCD [30] effect, except that the effect is practiced in minority ions rather than electrons. Thus, reflecting minority ions through a one-way ponderomotive well similarly drives current with potentially higher efficiency.

It remains, however, to identify how the effect might be implemented in a plasma device in a practical manner. First, it remains to identify suitable plasma waves that can be excited in confinement devices of interest, producing a very localized, intense rf field, so that nearly all particles in a flux tube can be reflected. Second, the fields must change sharply enough compared to the thermal gyroradius, which makes the proposed scheme more suitable for operating on ions rather than electrons. However, note that the optimized arrangement tolerates larger $\Lambda$, whether the method involvels ion or electron acceleration, and so operates with more easily implementable magnetic field gradients. Nonetheless, we expect that the new method is more likely to find use on linear and, perhaps, non-fusion plasma devices. 


\section{NUMERICAL RESULTS}

In this section, we present the results of numerical calculations of single-particle orbits for various field profiles and show that these calculations support our theoretical predictions.

\section{A. Smooth fields with even $\varepsilon(z)$}

Particle orbits were traced for the fields

$$
b(z)=1+\frac{z}{L_{B}}, \quad \varepsilon(z)=a \exp \left(-\frac{z^{2}}{L_{E}^{2}}\right),
$$

with parameters given in Table I and $v_{z, 0} \sim \hat{v}$. First, consider particles which travel from the strong-field side of the barrier $(\Omega>\omega)$. The numerical results are shown in Fig. 4, 5. All particles are seen to transmit through the resonance region, experience transverse cyclotron heating near $z=0$, and undergo adiabatic diamagnetic acceleration afterwards. The less is the initial longitudinal velocity, the more time a particle spends near the resonance. Thus, the more transverse energy it gains, and the stronger diamagnetic acceleration follows the resonant interaction. The asymptotic analytic approximation for $\Delta \mathcal{E}_{\perp}$ is found to be in reasonable agreement with numerical results even for $v_{z, 0} \sim \hat{v}$ (Fig. 6).

Consider now particles, which travel from the weak-field side of the barrier $(\Omega<\omega)$. The numerical results are shown in Fig. 7, 8. Particles with $v_{z, 0} \ll \hat{v}$ adiabatically reflect from the barrier without substantial gain of transverse energy. Others penetrate the region of resonant interaction and experience transverse heating near $z=0$. Since they gain a finite magnetic moment, behind the barrier these particles continue to "feel" decelerating diamagnetic force. To clarify the heating process for reflecting particles, we also present three-dimensional (3D) figures, which show both particle trajectory $z(\tau)$ and the evolution of transverse energy $\mathcal{E}_{\perp}$ (Fig. 9).

The energy constraint, which connects the longitudinal and transverse energy gain, has also been checked numerically. The error $\delta \mathcal{E} \equiv \Delta \mathcal{E}_{||}-\left(1-b_{0}\right) \Delta \mu$ was found to be less than $10^{-3}$ in units $a^{2} \sqrt{\Lambda}$. Thus, $\delta \mathcal{E} \ll \Phi_{\max }$ for all (both for reflected and transmitted) particles, as predicted.

\section{B. Smooth fields with odd $\varepsilon(z)$}

Particle orbits were traced for the fields

$$
b(z)=1+\frac{z}{L_{B}}, \quad \varepsilon(z)=a z \exp \left(-\frac{z^{2}}{L_{E}^{2}}\right),
$$

with parameters given in Table I and $v_{z, 0} \sim \hat{v}$. From our analytical model, it is expected that in such a field configuration particles should not get heated, as the condition (50) is satisfied within the resonance region with good accuracy. It is also predicted that such a heating-free barrier must yield completely reversible particle motion and have symmetric reflecting properties.

These expectations are confirmed by our numerical results. In Fig. 10, the phase portrait of the longitudinal particle motion shows that the established ponderomotive potential is indeed reversible at all $z$. The reflection properties of the 


\begin{tabular}{c|c}
\hline \hline & \\
$L_{B}$ & 100 \\
$L_{E}$ & 10 \\
$a$ & $10^{-3}$ \\
$\hat{v}=a^{4 / 5} L_{B}^{1 / 5}$ & 0.01 \\
$\Lambda=L_{B} / \hat{v}$ & $10^{4}$ \\
$\bar{z}=\sqrt{L_{B} \hat{v}}$ & 1 \\
$\mu_{0}$ & 0 \\
\hline \hline
\end{tabular}

TABLE I: Parameters for numerical calculations of particle motion in smooth fields (124), (125).

barrier are symmetric, meaning that if a particle traveling with initial velocity $v_{z, 0}$ is transmitted (reflected) through the barrier, a particle with initial velocity $-v_{z, 0}$ would also be transmitted (reflected) by the barrier, assuming equal $\mu_{0}$ for the two. In Fig. 11, 12, 13, it is shown that the energy gain for both transmitted and reflected particles incident from both sides is small. In general, such an rf field behaves as an adiabatic barrier, as the rf field has a small amplitude close to the cyclotron resonance.

\section{Abrupt fields}

Particle orbits were traced for the fields

$$
\begin{aligned}
& b(z)=1+\frac{1}{\sqrt{\Lambda}} \tanh \left(\frac{z}{\Delta z}\right) \\
& \varepsilon(z)=a \exp \left(-\frac{z^{2}}{L_{E}^{2}}\right)
\end{aligned}
$$

with parameters given in Table II and $v_{z, 0} \sim \hat{v}$, which simulates a single-boundary field configuration (84) with a continuous $\varepsilon(z)$ and $\alpha=1$ (see also Fig. 20). The following theoretical predictions (see Appendix B) were validated numerically: The minimum particle velocity sufficient to allow a particle to reach the boundary is $v_{z, 0} \approx 0.5 \hat{v}$ [Eq. (B3) yields $v_{z, 0}=\frac{1}{2} \hat{v}$, to transmit through the boundary $-v_{z, 0} \approx 0.87 \hat{v}$ [Eq. (B7) yields $v_{z, 0}=\frac{\sqrt{3}}{2} \hat{v}$, to transmit through the whole barrier $-v_{z, 0} \approx \hat{v}$ [Eq. (B8) yields $v_{z, 0}=\hat{v}$ ] - see Fig. 14, 15. The transverse energy of transmitted particles is approximately the same for all initial conditions [compare with Eq. (86)] - see Fig. 16. Minor variations in final values of $\mathcal{E}_{\perp}$ for different $v_{z, 0}$ result from weak nonadiabaticity of the particle motion at $|z| \gtrsim \Delta z$. (Despite $b(z)$ is uniform far away from $z=0$, the rf field inhomogeneity is sufficient to cause nonadiabatic effects, as long as $b$ remains close to unity. The effect is discussed in details in Refs. [16, 17].) The proportionality (82a) between the changes in the longitudinal and the transverse energies has also been checked numerically, and the error $\Delta \mathcal{E}_{\|}-\left(1-b_{0}\right) \Delta \mu$ was found to be much less than $\Phi_{\max }$. 


\begin{tabular}{c|c}
\hline \hline & \\
$\Delta z$ & $10^{-4}$ \\
$L_{E}$ & 10 \\
$a$ & $10^{-3}$ \\
$\Lambda$ & $10^{4}$ \\
$\hat{v}=a \Lambda^{1 / 4}$ & 0.01 \\
$\mu_{0}$ & 0 \\
& \\
\hline \hline
\end{tabular}

TABLE II: Parameters for numerical calculations of particle motion in abrupt fields (126), (127).

Particle orbits were also traced in abrupt field profiles with an odd $\varepsilon(z)$ :

$$
\begin{aligned}
& b(z)=1+\frac{1}{\sqrt{\Lambda}} \tanh \left(\frac{z}{\Delta z}\right) \\
& \varepsilon(z)=-a \exp \left(-\frac{z^{2}}{L_{E}^{2}}\right) \tanh \left(\frac{z}{\Delta z}\right),
\end{aligned}
$$

with parameters given in Table II and $v_{z, 0} \sim \hat{v}$, which simulates a single-boundary field configuration (111), (84) with an abrupt $\varepsilon(z)$ yielding the condition (88) and $\alpha=1$ (Fig. 3). As predicted analytically, the transverse heating is seen to be of the order of $\Delta \mu \lesssim a^{2}$. The theoretical predictions on the longitudinal momentum gain [Eq. (116)] also coincide with the results of numerical computations shown in Fig. 17.

Particle motion was also computed in a circularly-polarized rf field with field profiles (127). The phase portrait for the longitudinal motion is shown in Fig. 18. As predicted for this case (see Appendix A), the transverse heating is damped (Fig. 19), and, correspondingly, the established ponderomotive potential appears to be completely reversible for all $z$.

\section{SUMMARY}

In this paper, we showed that the action of an $\mathrm{rf}$ field on particles traveling along a dc magnetic field near the cyclotron resonance is similar to what essentially constitutes an operation of a Maxwell demon. Namely, an rf field can adiabatically reflect particles incident on the weak-field side of the ponderomotive barrier $(\Omega<\omega)$, while transmitting those incident on its strong-field side $(\Omega>\omega)$. Unlike the true Maxwell demon (prohibited by the second law of thermodynamics), an asymmetric barrier unavoidably heats particles, as they transit through the cyclotron resonance. Under fairly general assumptions about the field structure and polarization, an analytical model of transverse heating and longitudinal acceleration of particles shows fundamental correlations between the two effects.

A major result of this work is that we showed how to optimize the asymmetric ponderomotive current drive effect (APCD) for allowable transformations of a charged particle velocity by an rf field. In particular, we showed that an abrupt phase change in the rf field, coupled with an abrupt inhomogeneity of a dc field near the cyclotron resonance, gives a much higher efficiency for the APCD effect than that calculated in Ref. [11]. The latter is due to the fact that 
in such a configuration, energetically expensive transverse heating is replaced by a less energy-consuming heating of particles in the longitudinal direction. The method of optimization should be applicable to other applications of the asymmetric ponderomotive barrier as well, where the amount and direction of unavoidable heating of the transiting particles may be important.

\section{ACKNOWLEDGMENTS}

The work is supported by DOE contract DE-AC0276-CHO3073.

\section{APPENDIX A: EFFECT OF POLARIZATION}

To study how the rf polarization affects Eqs. (45)-(46) and Eqs. (82), (83), let us first consider the case when particles are driven by an rf field of circular polarization with the vector potential

$$
\mathbf{A}_{\mathrm{rf}}=\varepsilon(z)\left(\mathbf{x}^{0} \cos \tau-\mathbf{y}^{0} \sin \tau\right)
$$

Since the rf field is then given by

$$
\begin{aligned}
& \mathbf{E}_{\mathrm{rf}}=\varepsilon(z)\left(\mathbf{x}^{0} \sin \tau+\mathbf{y}^{0} \cos \tau\right), \\
& \mathbf{B}_{\mathrm{rf}}=\varepsilon^{\prime}(z)\left(\mathbf{x}^{0} \sin \tau+\mathbf{y}^{0} \cos \tau\right),
\end{aligned}
$$

the motion equations can be put in the following form:

$$
\begin{aligned}
& \dot{v}_{x}=v_{y} b+y v_{z} \frac{b^{\prime}}{2}-\frac{d}{d \tau}(\varepsilon \cos \tau), \\
& \dot{v}_{y}=-v_{x} b-x v_{z} \frac{b^{\prime}}{2}+\frac{d}{d \tau}(\varepsilon \sin \tau), \\
& \dot{v}_{z}=\left(x v_{y}-y v_{x}\right) \frac{b^{\prime}}{2}+\varepsilon^{\prime} \Re\left(w e^{i \tau}\right) .
\end{aligned}
$$

The average longitudinal force now yields Eq. (56), with

$$
\begin{aligned}
\langle U\rangle=\varepsilon \Re G, & G=\left\langle w e^{i \tau}\right\rangle, \\
\left\langle\Delta \mathcal{F}_{1}\right\rangle=-\varepsilon \Re G^{\prime}, & \left\langle\Delta \mathcal{F}_{2}\right\rangle=(b-1) \mathcal{E}_{\mathrm{rf}}^{\prime},
\end{aligned}
$$

where averaging is performed only over the initial gyrophase $\phi_{0}$. In the leading-order approximation, one gets

$$
\dot{w}+i b w-\frac{\dot{b}}{2 b} w=-\frac{d}{d \tau}\left(\varepsilon e^{-i \tau}\right),
$$

and thus, approximately, $w_{\mathrm{rf}}=i h e^{-i \chi-i \tau}$. Expressing $\mathcal{E}_{\mathrm{rf}}$ and $G$ in terms of $h$ and $\chi$, one obtains

$$
\mathcal{E}_{\mathrm{rf}}=\frac{|h|^{2}}{2}, \quad G=i h e^{-i \chi}
$$

and thus

$$
\varepsilon\left\langle G^{\prime}\right\rangle=i v_{z}\left|h^{\prime}\right|^{2}-(1-b) h h^{\prime *} .
$$


Consequently,

$$
\left\langle\Delta \mathcal{F}_{1,2}\right\rangle= \pm(1-b) \frac{d}{d z} \frac{|h|^{2}}{2}
$$

which yields Eq. (65). One can see that, in case of circularly polarized field, the derivation of Eq. (65) does not require averaging over the rf phases of particles and thus automatically applies to both smooth and abrupt fields.

Finally, one can formulate the obtained results as follows: In case of an rf field with circular polarization (A1), the average of the longitudinal force over the initial gyrophase $\phi_{0}$ is given by Eq. (76), where $\Delta \mu_{\mathrm{rf}} \equiv\left\langle\mathcal{M}_{\mathrm{rf}}\right\rangle(\infty)$ and $\langle U\rangle$ are given by

$$
\Delta \mu_{\mathrm{rf}} \approx \frac{1}{2}\left\langle|H|^{2}\right\rangle, \quad\langle U\rangle=-v_{z} \Im\left(h h^{*}\right)
$$

both for smooth and abrupt fields. This statement is true for a rotationally symmetric dc magnetic field (8), but is violated for an asymmetric field (e.g., $\left.\mathbf{B}_{0}=-x b^{\prime} \mathbf{x}^{0}+b \mathbf{z}^{0}\right)$. In the latter case, Eq. (76) can still be derived, yet that requires additional averaging over the rf phase, as discussed in Sec. VII and VIII.

One can see that, with minor reservations, the cases of linear and resonant circular polarization are equivalent, and thus the proposed calculations cover all imaginable cases of interest. Namely, in the general case of an elliptically polarized rf field, particles would primarily interact with the resonant circularly polarized harmonic, for which the presented analysis remains fully applicable.

\section{APPENDIX B: REFLECTION AND TRANSMISSION IN ABRUPT FIELD WITH STRONG TRANSVERSE HEATING}

Consider an abrupt-field configuration (84) with $b_{1,2}$ given by Eq. (111) and smooth rf field profile $\varepsilon \equiv \varepsilon_{1}=\varepsilon_{2}$ (Fig. 20). Using the expressions for $\phi_{0}$-averaged quantities

$$
\begin{aligned}
& \langle\Delta \mu\rangle_{\phi_{0}}=\frac{(1+\alpha)^{2}}{8 \alpha^{2}} \varepsilon^{2} \Lambda+\Delta \mu_{b}+\mathcal{O}\left(\varepsilon^{2}\right), \\
& \langle Q\rangle_{\phi_{0}}=-\Delta \mu_{b}+\mathcal{O}\left(\varepsilon^{2}\right),
\end{aligned}
$$

one can obtain the condition for particle transmission through the resonance. Assume $\mu_{0}=0$ for clarity. A particle is reflected purely by the ponderomotive force before reaching the resonance region if

$$
\frac{v_{z, 0}}{\hat{v}}<\frac{1}{2 \sqrt{\alpha}}
$$

where $\hat{v}^{2}=\varepsilon^{2} \sqrt{\Lambda}$. A weaker condition is imposed on $v_{z, 0}$ for reflection by the "boundary" itself: At location close to $z=0$ before colliding with the boundary, the particle longitudinal energy is given by

$$
\mathcal{E}_{\|}=\mathcal{E}_{\|, 0}-\Phi_{1}, \quad \Phi_{1}= \pm \frac{\hat{v}^{2}}{8 \alpha}
$$

The transmitted particle energy changes over the boundary according to $\left[\mathcal{E}_{\|}\right]=-\left[\mathcal{E}_{\perp}\right]$, where

$$
\left[\mathcal{E}_{\perp}\right]= \pm \frac{1+\alpha}{8 \alpha^{2}} \hat{v}^{2}
$$


as one can obtain straightforwardly from Eqs. (91). Since $\mathcal{E}_{\|}>0$, for a transmitted particle one must have

$$
\left(\frac{v_{z, 0}}{\hat{v}}\right)^{2}> \pm \frac{2 \alpha+1}{4 \alpha^{2}} .
$$

Particles traveling from the strong-field side are always transmitted, as seen from Eq. (B6) with the minus sign.

Those, which travel backwards, are reflected if

$$
\frac{v_{z, 0}}{\hat{v}}<\frac{\sqrt{2 \alpha+1}}{2 \alpha} .
$$

Note also that the violation of inequality (B7) does not necessarily prevent a particle from reflection by the decelerating ponderomotive force behind the resonance. To eliminate this possibility, it is necessary to have

$$
\frac{v_{z, 0}}{\hat{v}}>\frac{\alpha+1}{2 \alpha} .
$$


[1] A. V. Gaponov and M. A. Miller, Sov. Phys. JETP 7, 168 (1958).

[2] H. Motz and C. J. H. Watson, Advances in Electronics 23, 153 (1967).

[3] I. Y. Dodin and N. J. Fisch, Approximate integrals of rf-driven particle motion in magnetic field, to be appear in J. Plasma Phys. (2005).

[4] P. K. Shukla and R. Schlickeiser, Phys. Plasmas 10, 1523 (2003).

[5] A. Guglielmi and R. Lundin, J. Geophys. Res. 106, 13219 (2001).

[6] N. C. Lee and G. K. Parks, Geophys. Res. Lett. 23, 327 (1996).

[7] E. S. Weibel, Phys. Rev. Lett. 44, 377 (1980).

[8] J. R. Ferron, N. Hershkowitz, R. A. Breun, S. N. Golovato, and R. Goulding, Phys. Rev. Lett. 51, 1955 (1983).

[9] Y. Yasaka and R. Itatani, Phys. Rev. Lett. 56, 2811 (1986).

[10] E. V. Suvorov and M. D. Tokman, Fizika Plazmy 14, 950 (1988) [in Russian]; A. G. Litvak, A. M. Sergeev, E. V. Suvorov, M. D. Tokman, and I. V. Khazanov, Phys. Fluids B 5, 4347 (1993).

[11] N. J. Fisch, J. M. Rax, and I. Y. Dodin, Phys. Rev. Lett. 91, 205004 (2003).

[12] E. Canobbio, Nuclear Fusion 9, 27 (1969).

[13] A. J. Lichtenberg and H. L. Berk, Nuclear Fusion 15, 999 (1975).

[14] T. Consoli and R. B. Hall, Nuclear Fusion 3, 237 (1963).

[15] T. Hatori and T. Watanabe, Nuclear Fusion 15, 143 (1975).

[16] G. Dimonte, B. M. Lamb, and G. J. Morales, Plas. Phys. 25, 713 (1983).

[17] B. M. Lamb, G. Dimonte, and G. J. Morales, Phys. Fluids 27, 1401 (1984).

[18] J. J. Martinell, Rev. Mex. Fis. 48, 239 (2002).

[19] H. P. Eubank, Phys. Fluids 12, 234 (1969).

[20] P. B. Parks and F. B. Marcus, Nuclear Fusion 21, 1207 (1981).

[21] E. M. Lifshitz and L. P. Pitaevskii, Physical Kinetics (Pergamon Press, New York, 1981).

[22] H. Ye and P. J. Morrison, Phys. Fluids B 4, 771 (1992).

[23] C. S. Gardner, Phys. Fluids B 6, 839 (1963).

[24] N. J. Fisch and J. M. Rax, Phys. Fluids B 5, 1754 (1993).

[25] I. Y. Dodin and N. J. Fisch, Variational formulation of the Gardner's restacking algorithm, submitted to J. Math. Phys. (2004).

[26] A. Kildal, Contrib. Plasma Phys. 39, 349 (1999).

[27] B. Hafizi and R. E. Aamodt, Phys. Fluids 30, 3059 (1987).

[28] T. Ohkawa, General Atomic Co. Report A13847 (1976).

[29] N. J. Fisch, Phys. Rev. Lett. 41, 873 (1978).

[30] N. J. Fisch and A. H. Boozer, Phys. Rev. Lett. 45, 720 (1980).

[31] N. J. Fisch, Rev. Mod. Phys. 59, 175 (1987).

[32] N. J. Fisch, Nuclear Fusion 21, 15 (1981). 


\section{LIST OF CAPTIONS}

1. Schematic of an asymmetric ponderomotive barrier with an $\mathrm{rf}$ electric field having a maximum at $z=0$, where the local cyclotron frequency $\Omega=e B_{0} / m c$ equals the $\mathrm{rf}$ field frequency $\omega$. Ponderomotive potential $\Phi(z)$ is singular and changes its sign over the cyclotron resonance. The average longitudinal Lorentz force is directed the same way to both sides of the resonance and drags a charged particle towards weaker dc magnetic field $\mathbf{B}_{0}$.

2. Ponderomotive potential in a smooth field configuration $\left(L_{B}, L_{E} \gg v_{z} / \omega\right)$ : in the resonance region (shaded), where the ponderomotive approximation does not hold, the rf electric field is approximately constant, and the dc magnetic field can be approximated with a linear function of $z\left(\Omega / \omega \approx 1+z / L_{B}\right)$. The characteristic width of the resonance region is $\bar{z}=\sqrt{L_{B} v_{z} / \omega}$; the characteristic height of the ponderomotive barrier is about $\Phi(\bar{z})$.

3. Schematic of the abrupt field configuration with reduced transverse heating: the rf electric field amplitude $\varepsilon(z)$ equals zero at the cyclotron resonance and satisfies Eq. (50) outside the resonance region. The dc magnetic field and the rf field change at the scale $\Delta z$ small compared to the longitudinal particle gyroradius $r_{g}\left(r_{g}=v_{z}\right.$ in dimensionless units).

4. Normalized transverse energy $\mathcal{E}_{\perp} / a^{2} \Lambda$ of particles incident on a smooth rf barrier from the strong-field side $(\Omega>\omega)$ versus $z$ for $v_{z, 0} / \hat{v}=0.5,0.75,1,1.5,2,3,4,5: b(z)$ and $\varepsilon(z)$ yield Eq. (124) with parameters given in Table I. Larger energy gain corresponds to smaller $\left|v_{z, 0}\right|$.

5. Phase portrait for the longitudinal motion of particles incident on a smooth rf barrier from the strong-field side $(\Omega>\omega)$ : normalized longitudinal velocity $v_{z} / \hat{v}$ versus $z$ (same parameters than for Fig. 4). Larger acceleration corresponds to smaller $\left|v_{z, 0}\right|$.

6. Normalized transverse energy gain $\mathcal{E}_{\perp} / a^{2} \Lambda$ of particles incident on a smooth rf barrier from the strong-field side $(\Omega>\omega)$ versus $v_{z, 0} / \hat{v}$ (same parameters than for Fig. 4): numerical (solid) and asymptotical analytical approximation (dashed) for $v_{z, 0} / \hat{v} \gg 1$.

7. Normalized transverse energy $\mathcal{E}_{\perp} / a^{2} \Lambda$ of particles incident on a smooth rf barrier from the weak-field side $(\Omega<\omega)$ versus $z$ for $v_{z, 0} / \hat{v}=0.2,0.5,1,2,3,4,5$ (same parameters than for Fig. 4).

8. Phase portrait for the longitudinal motion of particles incident on a smooth rf barrier from the weak-field side $(\Omega<\omega)$ : normalized longitudinal velocity $v_{z} / \hat{v}$ versus $z$ (same parameters than for Fig. 4).

9. Particle motion near a smooth cyclotron-resonant $\mathrm{rf}$ barrier. Axes: normalized transverse energy $\mathcal{E}_{\perp} / a^{2} \Lambda$ of a particle, its longitudinal location $z$, and time $\tau$. Separately shown is the projection of the 3D plot on the plane $\left(\tau, \mathcal{E}_{\perp}\right)$ (same parameters than for Fig. 4): (a) $v_{z, 0} / \hat{v}=0.2$ - adiabatic reflection by the $\mathrm{rf}$ field; (b) $v_{z, 0} / \hat{v}=0.5$ - slightly nonadiabatic reflection by the $\mathrm{rf}$ field; (c) $v_{z, 0} / \hat{v}=2$ - adiabatic diamagnetic reflection after nonadiabatic resonant heating.

10. Phase portrait of a cyclotron-resonant rf barrier produced by the fields given by Eq. (125) with parameters given in Table I: normalized longitudinal velocity $v_{z} / \hat{v}$ versus $z$. Established potential is reversible for all $z$. 
11. Normalized transverse energy $\mathcal{E}_{\perp} / a^{2} \Lambda$ of particles incident on a smooth rf barrier with an odd $\varepsilon(z)$ from the weak-field $(\Omega<\omega)$ side versus $z$ (same parameters than for Fig. 10): $v_{z, 0} / \hat{v}=3$ (for other values of $v_{z, 0} / \hat{v}$, plots are virtually congruent with the shown one, as motion is reversible).

12. Particle motion near a smooth cyclotron-resonant $\mathrm{rf}$ barrier with an odd profile $\varepsilon(z)$. Axes: normalized transverse energy $\mathcal{E}_{\perp} / a^{2} \Lambda$ of a particle, its longitudinal location $z$, and time $\tau$. Separately shown is the projection of the $3 \mathrm{D}$ plot on the plane $\left(\tau, \mathcal{E}_{\perp}\right)$ (same parameters than for Fig. 10): (a,b) $v_{z, 0} / \hat{v}= \pm 0.85$ - adiabatic reflection by the rf field of particles traveling from the weak- and the strong-field $(\Omega \lessgtr \omega)$ sides.

13. Particle motion near a smooth cyclotron-resonant $\mathrm{rf}$ barrier with an odd profile $\varepsilon(z)$. Axes: normalized transverse energy $\mathcal{E}_{\perp} / a^{2} \Lambda$ of a particle, its longitudinal location $z$, and time $\tau$. Separately shown is the projection of the $3 \mathrm{D}$ plot on the plane $\left(\tau, \mathcal{E}_{\perp}\right)$ (same parameters than for Fig. 10): (a,b) $v_{z, 0} / \hat{v}= \pm 0.9$ - adiabatic transmission of particles traveling from the weak- and the strong-field $(\Omega \lessgtr \omega)$ sides.

14. Phase portrait of a cyclotron-resonant rf barrier produced by the fields given by Eq. (126) with parameters given in Table II: normalized longitudinal velocity $v_{z} / \hat{v}$ versus $z\left(\right.$ solid $-v_{z, 0}>0$, dashed $\left.-v_{z, 0}<0\right)$. Particles traveling from the strong-field side $(\Omega>\omega)$ are all transmitted. Those, which travel from the weak-field side $(\Omega<\omega)$, are reflected adiabatically without reaching the boundary if $v_{z, 0}<\frac{1}{2} \hat{v}$, are reflected from the boundary - if $\frac{1}{2} \hat{v}<v_{z, 0}<\frac{\sqrt{3}}{2} \hat{v}$, transmit through the whole barrier - if $v_{z, 0}>\hat{v}$.

15. Particle motion near a single-boundary abrupt cyclotron-resonant rf barrier. Axes: normalized transverse energy $\mathcal{E}_{\perp} / a^{2} \Lambda$ of a particle, its longitudinal location $z$, and time $\tau$. Separately shown is the projection of the 3D plot on the plane $\left(\tau, \mathcal{E}_{\perp}\right)$ (same parameters than for Fig. 14): (a) $v_{z, 0} / \hat{v}=0.5$ - adiabatic reflection by the rf field of a particle traveling from the weak-field side $(\Omega<\omega)$ without reaching the boundary; (b) $v_{z, 0} / \hat{v}=1-$ particle reflection by the decelerating ponderomotive force behind the barrier; (c) $v_{z, 0} / \hat{v}=1.5$ - particle transmission.

16. Normalized transverse energy $\mathcal{E}_{\perp} / a^{2} \Lambda$ of particles incident on a single-boundary rf barrier (a) from the weakfield side $(\Omega<\omega)$ and (b) from the strong-field side $(\Omega>\omega)$ versus $z$ (same parameters than for Fig. 14): (a) $v_{z, 0} / \hat{v}=1.1,1.5$, (b) $v_{z, 0} / \hat{v}=0.3,0.5,0.7,1$. In wide range of initial velocities, particles gain approximately equal transverse energy $\mathcal{E}_{\perp} \approx \frac{1}{2} a^{2} \Lambda$, regardless of the sign of $v_{z, 0}$.

17. Normalized longitudinal velocity $v_{z} / v_{z, 0}$ of particles incident on a single-boundary heating-free rf barrier from the strong-field side $(\Omega>\omega)$ as a function of their initial rf phase $\tau_{0}$ : analytical (solid) and numerical (dotted) results; $b(z)$ and $\varepsilon(z)$ yield Eq. (127) with parameters given in Table II, $v_{z, 0}^{2}=\frac{1}{4} \hat{v}^{2}$, the reflection coefficient is $R=\frac{1}{2}$. Observed transverse heating is $\Delta \mu \lesssim a^{2}$.

18. Phase portrait of a cyclotron-resonant rf barrier produced by the fields given by Eq. (127) (circular polarization) with parameters given in Table II: normalized longitudinal velocity $v_{z} / \hat{v}$ versus $z$. Established potential is reversible for all $z$.

19. Normalized transverse energy $\mathcal{E}_{\perp} / a^{2} \Lambda$ of particles incident on an abrupt rf barrier with an odd $\varepsilon(z)$ from the 
weak-field side $(\Omega<\omega)$ versus $z$ (same parameters than for Fig. 18): $v_{z, 0} / \hat{v}=3$ (for other values of $v_{z, 0} / \hat{v}$, plots are congruent with the shown one, as motion is reversible).

20. Schematic of the abrupt field configuration with strong transverse heating: the rf electric field amplitude $\varepsilon(z)$ changes little over the resonance region. The dc magnetic field changes at the scale $\Delta z$ small compared to the longitudinal particle gyroradius $r_{g}\left(r_{g}=v_{z}\right.$ in dimensionless units). 


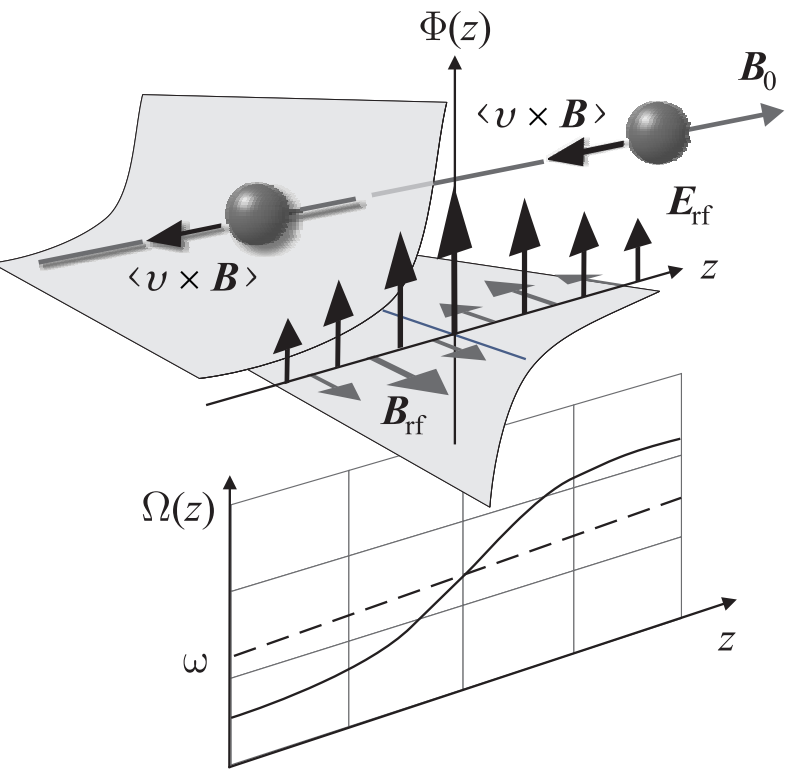

FIG. 1: 


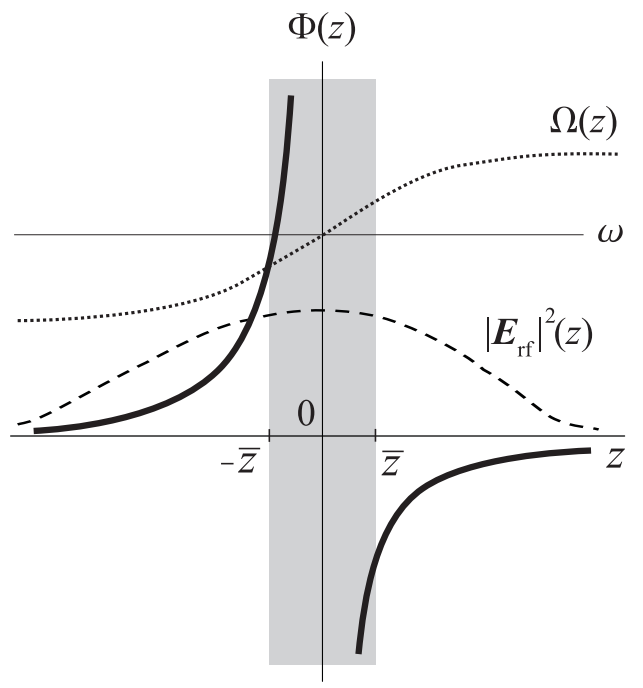

FIG. 2: 


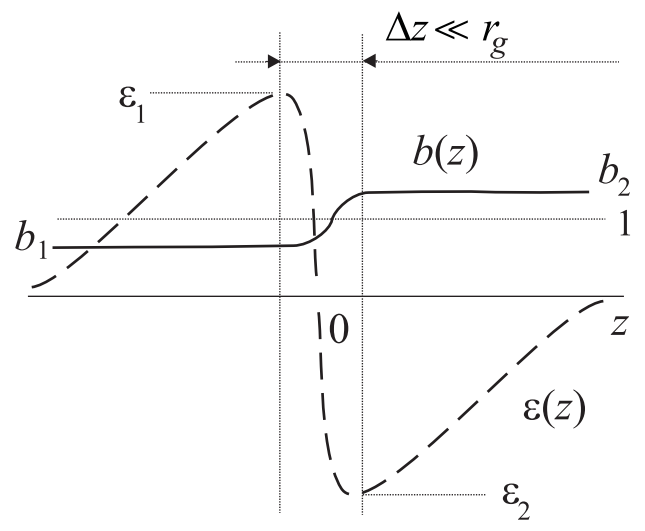

FIG. 3: 


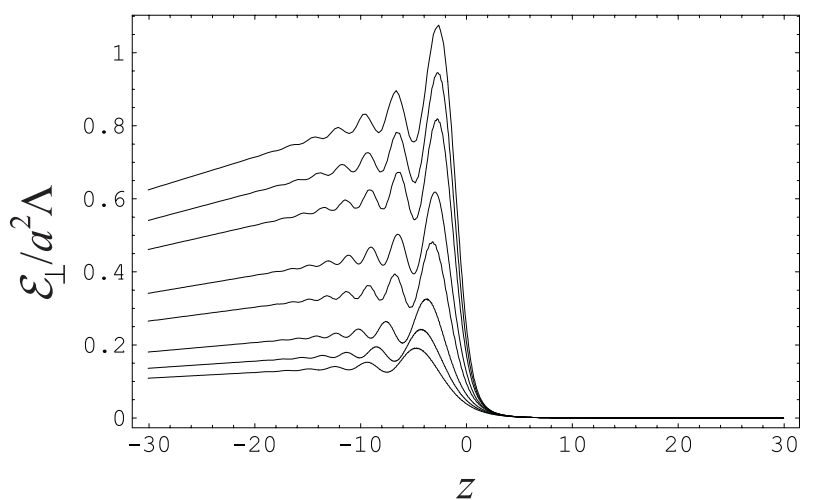

FIG. 4: 


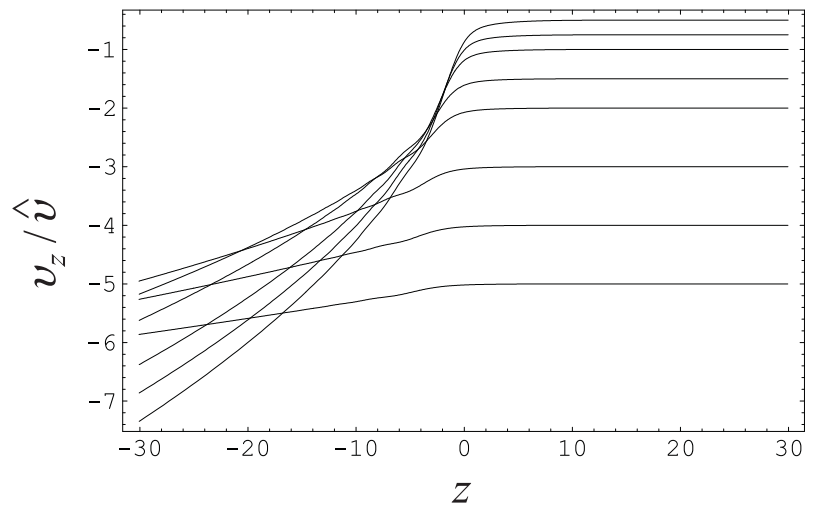

FIG. 5: 


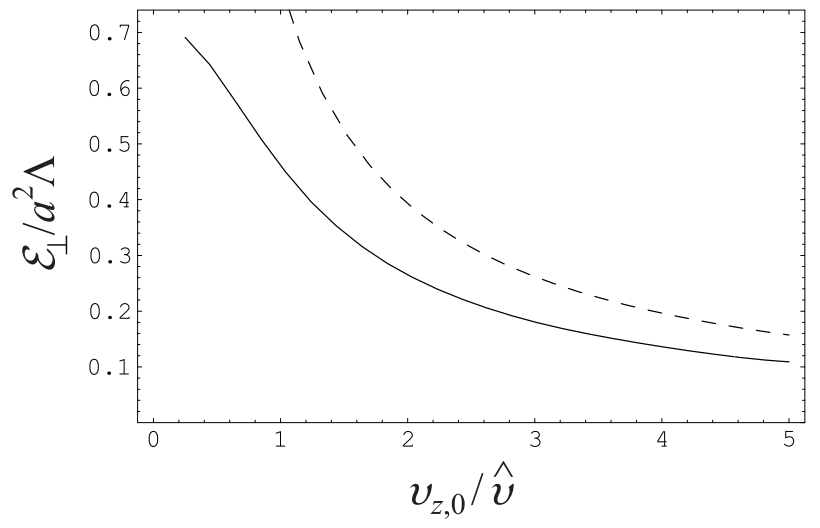

FIG. 6: 


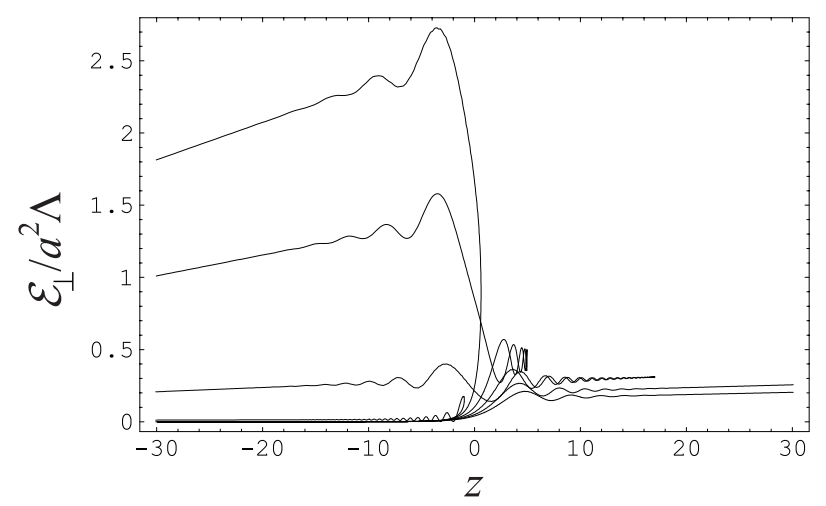

FIG. 7: 


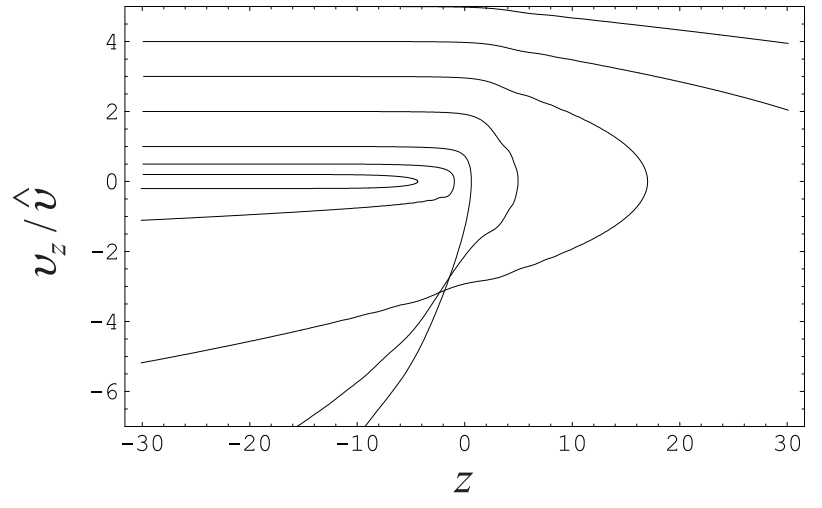

FIG. 8: 

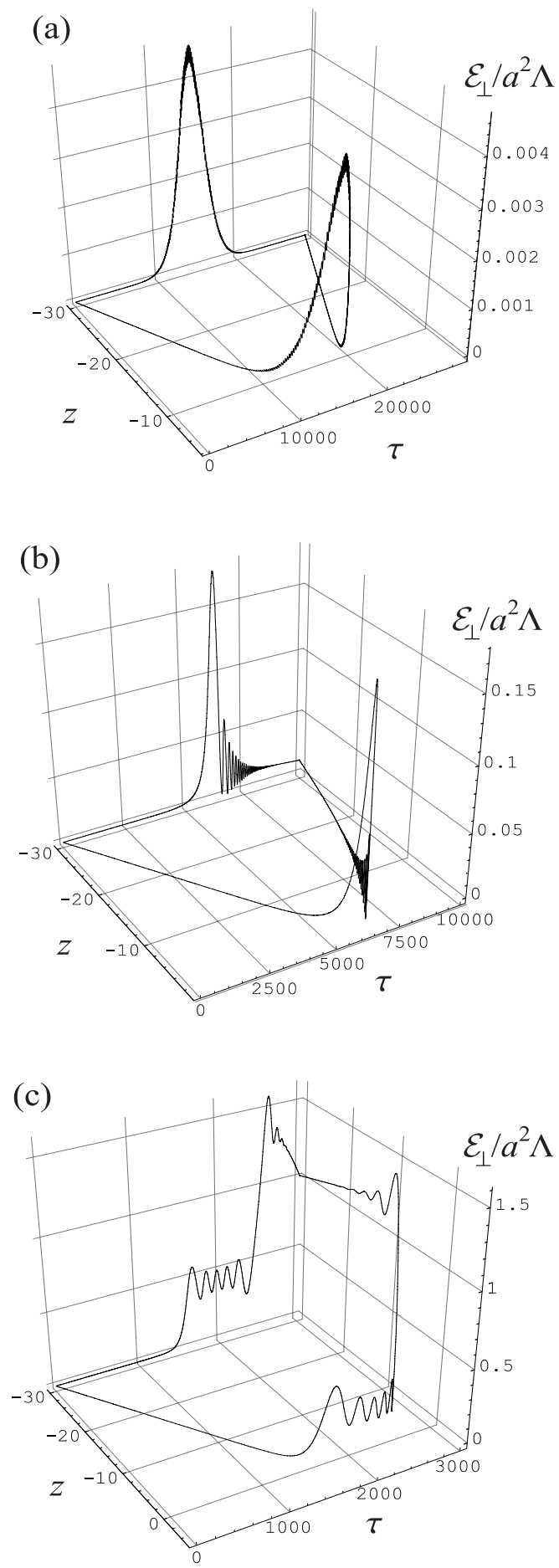

FIG. 9: 


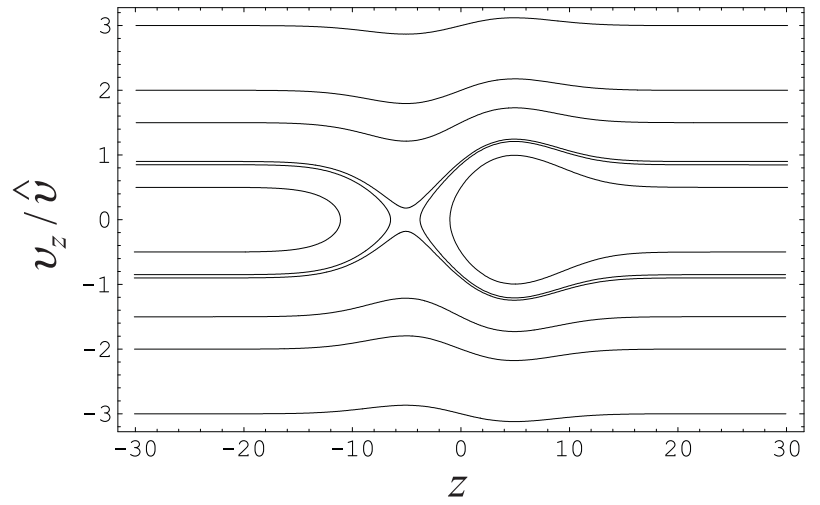

FIG. 10: 


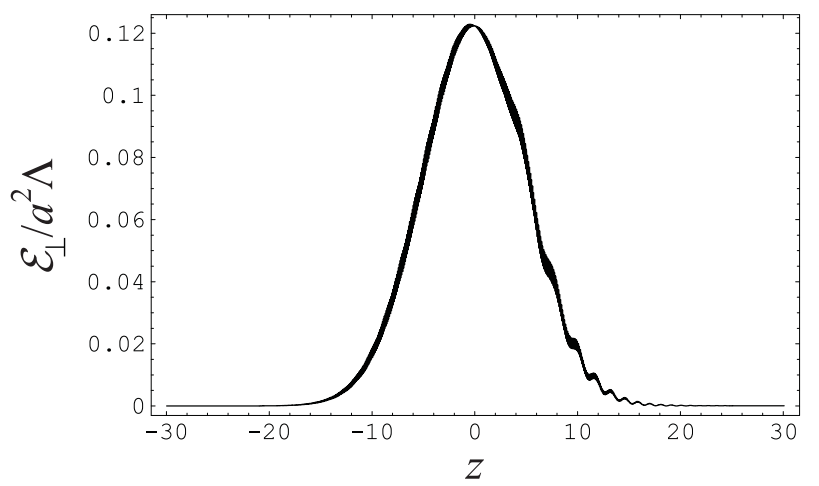

FIG. 11: 
(a)

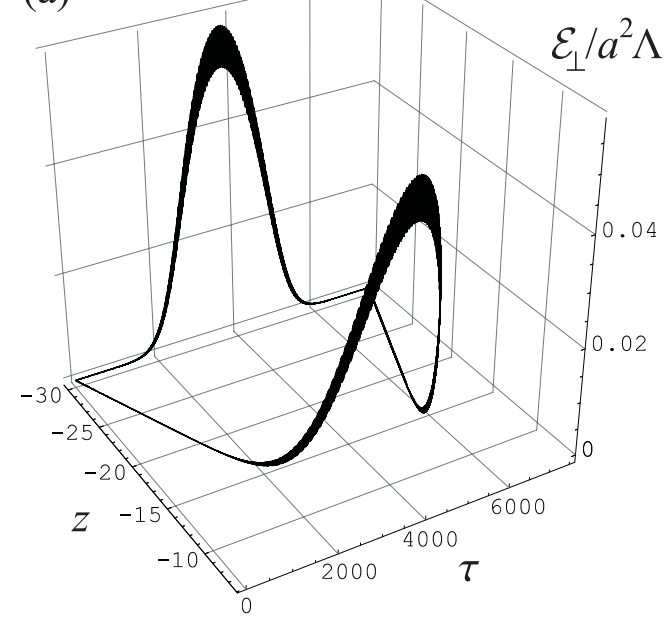

(b)

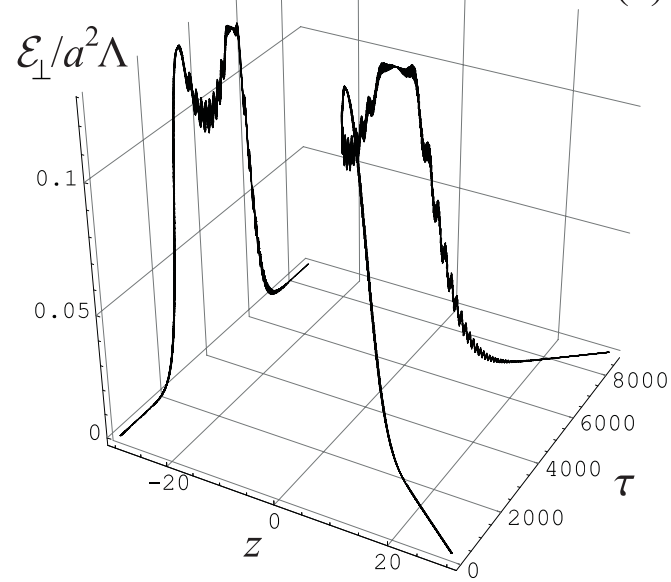

FIG. 12: 
(a)

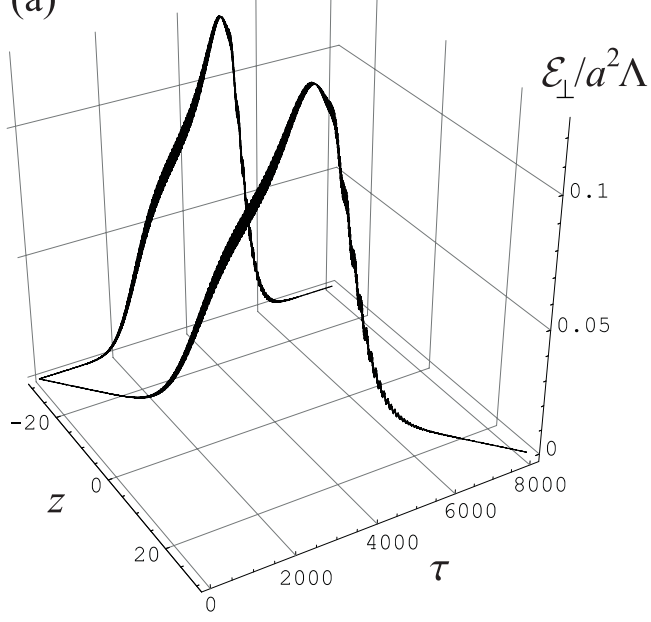

(b)

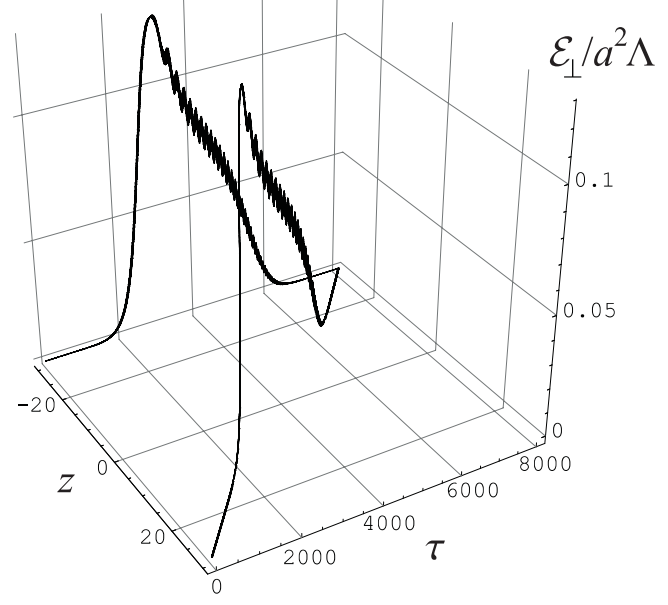

FIG. 13: 


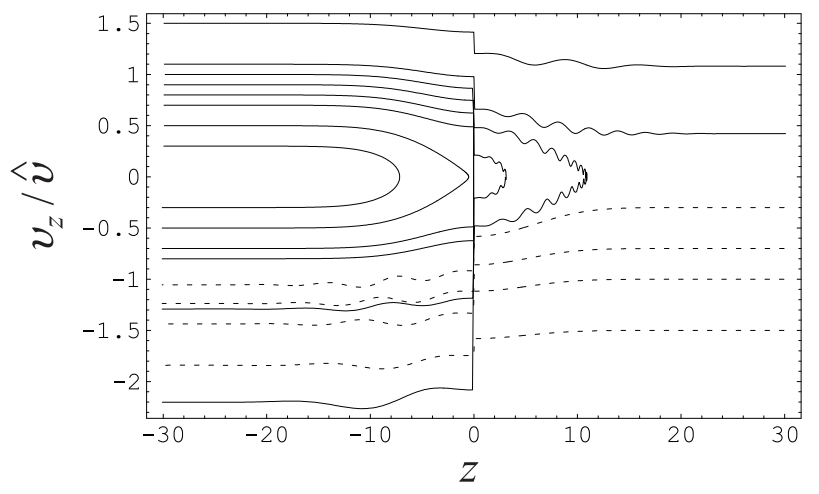

FIG. 14: 
(a)

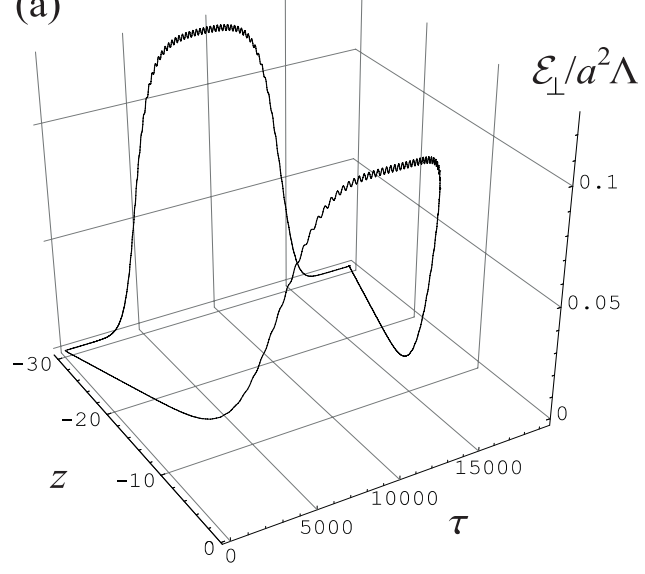

(b)
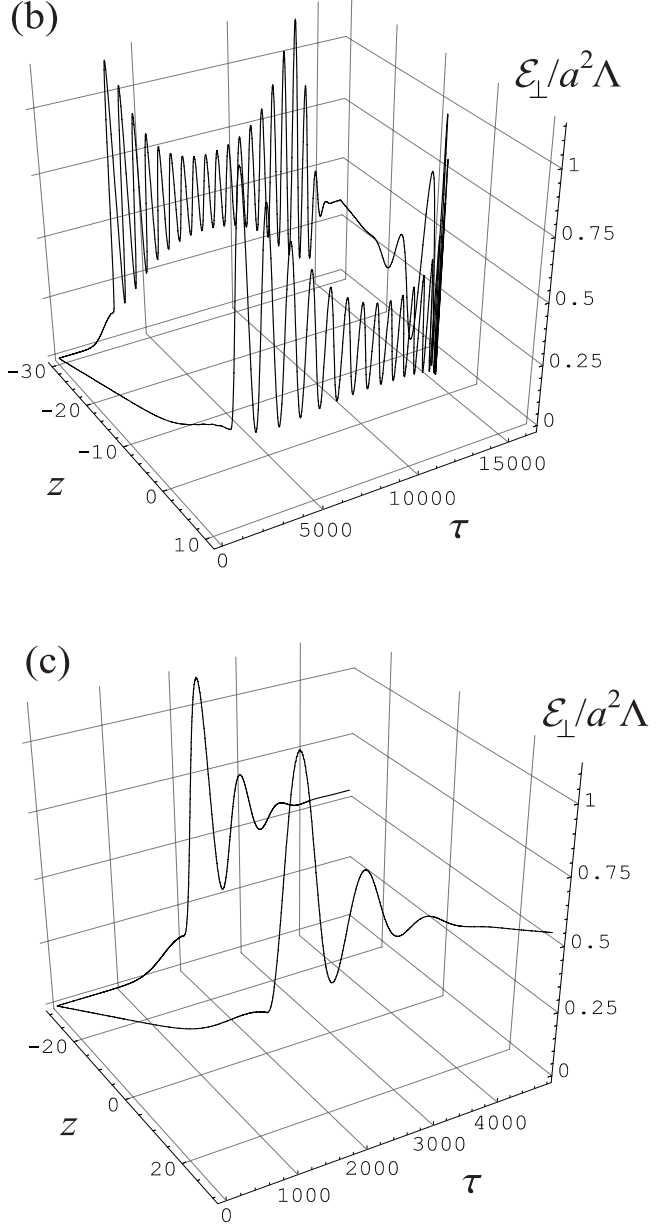

FIG. 15: 

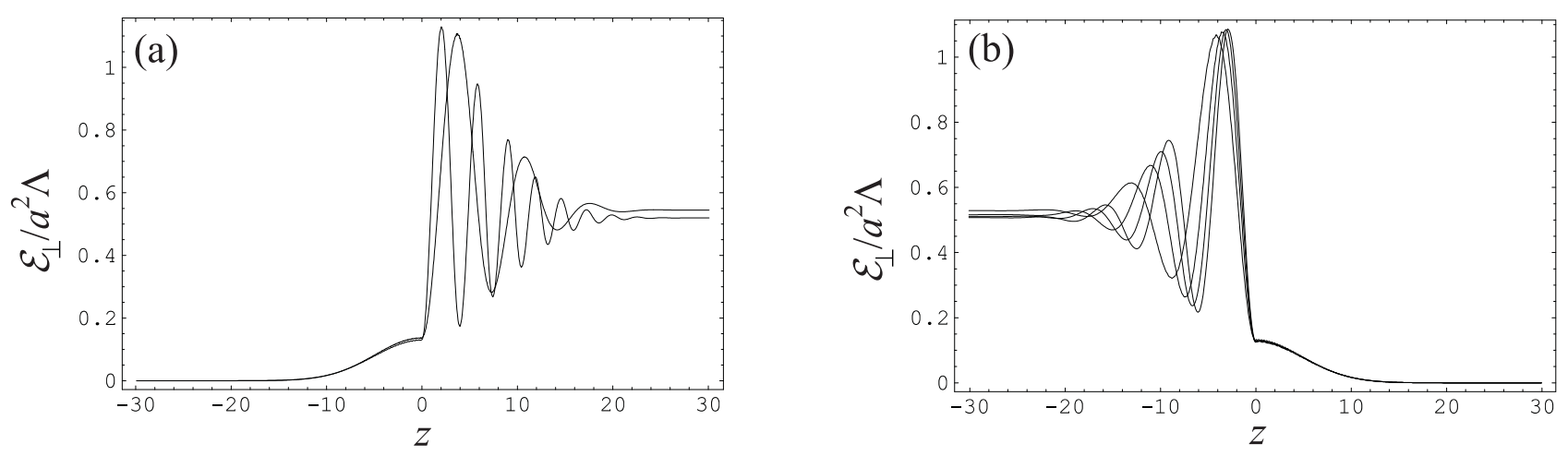

FIG. 16: 


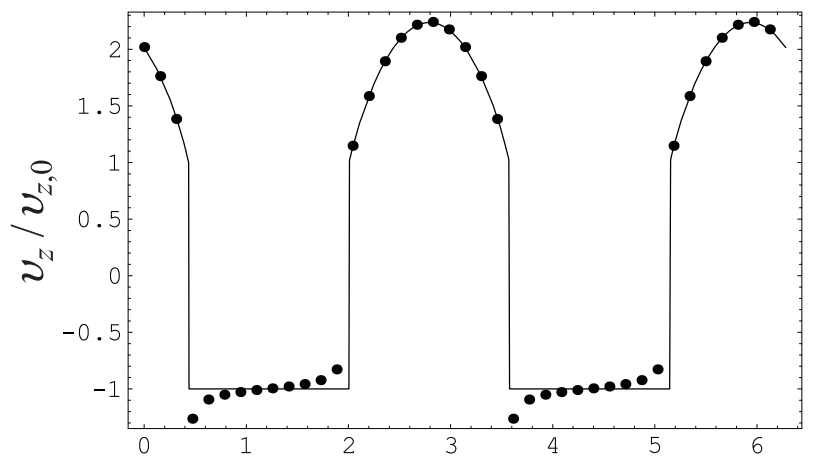

$\tau_{0}$

FIG. 17: 


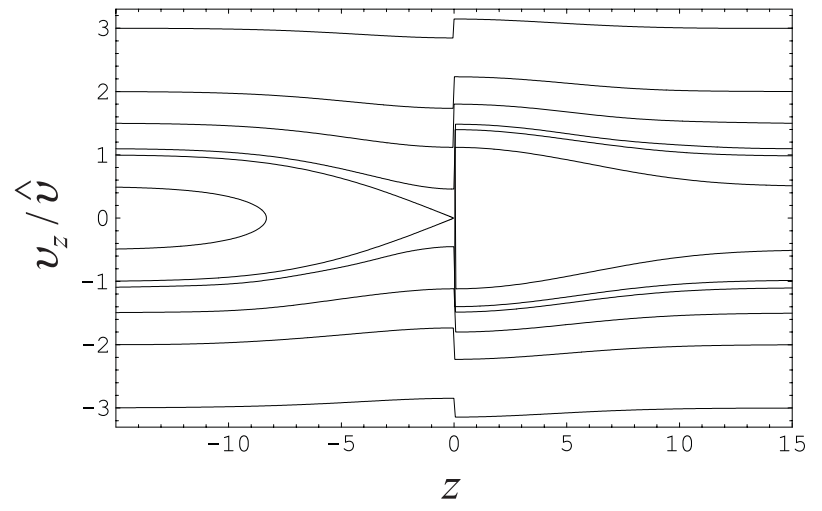

FIG. 18: 


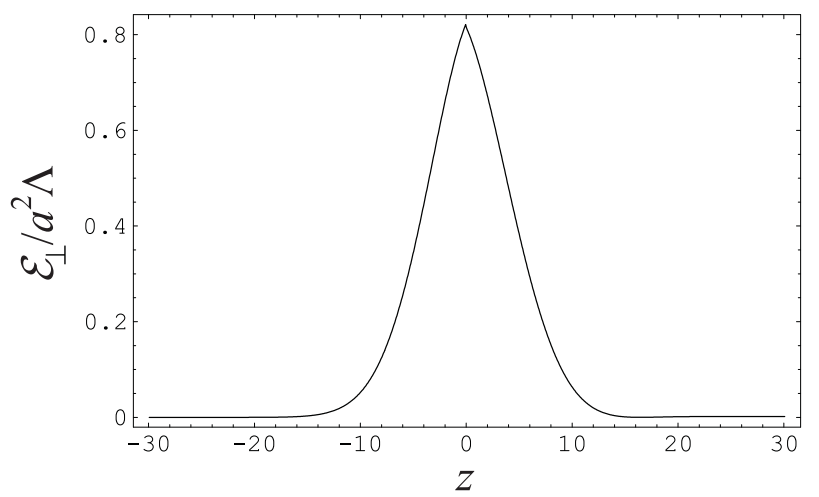

FIG. 19: 


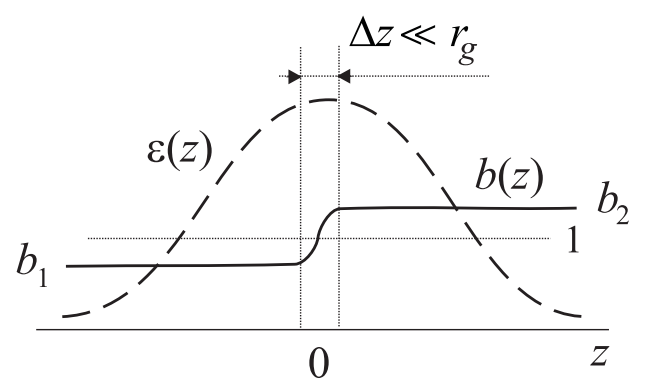

FIG. 20: 


\section{External Distribution}

Plasma Research Laboratory, Australian National University, Australia

Professor I.R. Jones, Flinders University, Australia

Professor João Canalle, Instituto de Fisica DEQ/IF - UERJ, Brazil

Mr. Gerson O. Ludwig, Instituto Nacional de Pesquisas, Brazil

Dr. P.H. Sakanaka, Instituto Fisica, Brazil

The Librarian, Culham Laboratory, England

Mrs. S.A. Hutchinson, JET Library, England

Professor M.N. Bussac, Ecole Polytechnique, France

Librarian, Max-Planck-Institut für Plasmaphysik, Germany

Jolan Moldvai, Reports Library, Hungarian Academy of Sciences, Central Research Institute for Physics, Hungary

Dr. P. Kaw, Institute for Plasma Research, India

Ms. P.J. Pathak, Librarian, Institute for Plasma Research, India

Ms. Clelia De Palo, Associazione EURATOM-ENEA, Italy

Dr. G. Grosso, Instituto di Fisica del Plasma, Italy

Librarian, Naka Fusion Research Establishment, JAERI, Japan

Library, Laboratory for Complex Energy Processes, Institute for Advanced Study, Kyoto University, Japan

Research Information Center, National Institute for Fusion Science, Japan

Dr. O. Mitarai, Kyushu Tokai University, Japan

Dr. Jiangang Li, Institute of Plasma Physics, Chinese Academy of Sciences, People's Republic of China

Professor Yuping Huo, School of Physical Science and Technology, People's Republic of China

Library, Academia Sinica, Institute of Plasma Physics, People's Republic of China

Librarian, Institute of Physics, Chinese Academy of Sciences, People's Republic of China

Dr. S. Mirnov, TRINITI, Troitsk, Russian Federation, Russia

Dr. V.S. Strelkov, Kurchatov Institute, Russian Federation, Russia

Professor Peter Lukac, Katedra Fyziky Plazmy MFF UK, Mlynska dolina F-2, Komenskeho Univerzita, SK-842 15 Bratislava, Slovakia

Dr. G.S. Lee, Korea Basic Science Institute, South Korea

Institute for Plasma Research, University of Maryland, USA

Librarian, Fusion Energy Division, Oak Ridge National Laboratory, USA

Librarian, Institute of Fusion Studies, University of Texas, USA

Librarian, Magnetic Fusion Program, Lawrence Livermore National Laboratory, USA

Library, General Atomics, USA

Plasma Physics Group, Fusion Energy Research Program, University of California at San Diego, USA

Plasma Physics Library, Columbia University, USA

Alkesh Punjabi, Center for Fusion Research and Training, Hampton University, USA

Dr. W.M. Stacey, Fusion Research Center, Georgia Institute of Technology, USA

Dr. John Willis, U.S. Department of Energy, Office of Fusion Energy Sciences, USA

Mr. Paul H. Wright, Indianapolis, Indiana, USA 
The Princeton Plasma Physics Laboratory is operated by Princeton University under contract with the U.S. Department of Energy.

\author{
Information Services \\ Princeton Plasma Physics Laboratory \\ P.O. Box 451 \\ Princeton, NJ 08543
}

Phone: 609-243-2750

Fax: 609-243-2751

e-mail: pppl_info@pppl.gov

Internet Address: http://www.pppl.gov 